\title{
Constante de Bers en genre 2
}

\section{Matthieu Gendulphe}

Received: 27 February 2007 / Revised: 19 January 2009 / Published online: 8 October 2010

(C) Springer-Verlag 2010

\begin{abstract}
We introduce a new tool, the contiguity graph, which enables us to deter-
\end{abstract} mine the Bers's constant in genus two.

Mathematics Subject Classification (2000) $\quad 30 \mathrm{~F} 45 \cdot 30 \mathrm{~F} 60$

\section{Table des matières}

0 Introduction . . . . . . . . . . . . . . . . . . . . . . . . . . . 919

1 Graphe de contiguïté . . . . . . . . . . . . . . . . . . . . . . . . . . . . . 922

2 Géométrie-Topologie associée à une géodésique séparante . . . . . . . . . . . . . . . . . . . . 927

3 Majoration de la longueur des arêtes du graphe de contiguïté . . . . . . . . . . . . . . . . . . 931

4 La 3-systole est la systole de la famille $\mathcal{G}_{\xi} \cup\{\xi\} \ldots \ldots$. . . . . . . . . . . . . . . . . . . . . . . . . . . 939

5 Existence et détermination du maximum global de sys 3 . . . . . . . . . . . . . . . . . . . . 944

\section{Introduction}

Nous considérons les surfaces de Riemann fermées de genre $g \geq 2$ munies de leur métrique hyperbolique de Poincaré.

L'auteur a été soutenu par la Swiss National Science Foundation (financement no. 200020-121506/1).

M. Gendulphe $(\varangle)$

Institut de Mathématiques de Bordeaux, Université Bordeaux 1, Bordeaux, France

e-mail: matthieu.gendulphe@unifr.ch

Present Address:

M. Gendulphe

Département de Mathématiques, Université de Fribourg, Chemin du Musée 23,

1700 Fribourg Pérolles, Switzerland 


\subsection{Constante de Bers}

Alors qu'il s'employait à compactifier l'espace des modules, Lipman Bers observa [6] que toute surface de Riemann possède une famille de $3 g-3$ géodésiques fermées simples et disjointes (une partition), dont les longueurs sont majorées par une constante optimale $B(g)$ dépendant seulement du genre $g$. La constante mise au jour par le mathématicien éponyme suscite plusieurs interrogations : Quelle est sa valeur ? Estelle réalisée par une surface ? Peut-on en donner une asymptotique ?

Dès 1980, Peter Buser majore $B(g)$ par $(6 g-4) \operatorname{arccosh}(2 \pi(g-1))$. En 1992, il parvient avec Mika Seppälä à un résultat plus précis [9], et la même année indique dans [8] une minoration de $B(g)$. Le tout donne une bonne idée du comportement asymptotique de la constante de Bers : $\sqrt{6 g}-2 \leq B(g)<21 g$.

Durant les années 90, Paul Schmutz Schaller s'intéresse aux fonctions de type systole sur les surfaces de Riemann, et se lance dans la recherche de leurs maxima, en particulier de $B(2)$ [15]. Dans un premier temps il s'attaque aux surfaces à bords, pour se diriger ensuite vers les surfaces fermées. La surface de Bolza se révèle alors l'unique maximum global de la systole et de la 2-systole en genre 2. Le cas de la 3-systole, bornée supérieurement par $B(2)$, s'avère plus hardu : Schmutz Schaller découvre deux maxima locaux et conjecture que ce sont les seuls.

À ce jour, aucune valeur de $B(g)$ n'est connue. La recherche de la constante de Bers en genre 2 se poursuit cependant $[13,14]$. Les méthodes de découpages de Schmutz Schaller n'ont toujours pas abouti, et la résolution de ce problème semble maintenant nécessiter l'élaboration d'autres techniques.

Dans cet article nous déterminons $B(2)$, plus précisément nous montrons :

Théorème La constante de Bers $B(2)$ est déterminée par $\cosh (B(2) / 12)=x_{0}$ où $x_{0}$ est l'unique solution supérieure à 1 de $32 x^{5}-32 x^{4}-24 x^{3}+24 x^{2}-1=0$, soit $\cosh (B(2) / 2) \approx 4,67$. La surface réalisant $B(2)$ est unique à isométrie près.

La surface réalisant $B(2)$ est une des deux surfaces 3-maximales construites dans [15]. La méthode mise en œuvre pour ce résultat, et décrite plus loin, permet la découverte d'un troisième maximum local de la 3-systole, infirmant ainsi la conjecture de Schmutz Schaller.

\subsection{Systole et $k$-systole}

La systole d'une surface de Riemann est la longueur de sa plus courte géodésique incontractile. Par abus de langage, on emploie le même mot pour les géodésiques la réalisant. En tant que minimum des fonctions longueur de géodésique, la systole définit une fonction continue sur l'espace de Teichmüller $\mathcal{T}_{g}$, invariante sous l'action du groupe modulaire $\operatorname{Mod}_{g}$. La caractérisation et la recherche de ses maxima locaux fut un sujet d'études très actif, dont émergea une théorie élégante [3,5], basée sur l'analogie avec l'invariant d'Hermite des réseaux euclidiens.

Schmutz Schaller généralise ainsi la systole [15] : considérant les familles de $k$ géodésiques fermées simples et disjointes, il associe à chacune d'elles une fonction 


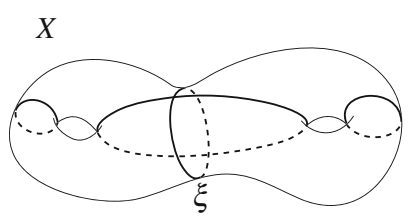

Fig. 1 Une partition en genre 2

longueur correspondant en tout point à la longueur de la plus grande géodésique :

$$
l_{\left\{\gamma_{1}, \ldots, \gamma_{k}\right\}}(M)=\sup \left(l_{\gamma_{1}}(M), \ldots, l_{\gamma_{k}}(M)\right) \quad\left(M \in \mathcal{T}_{g}\right) \text {. }
$$

Il introduit alors la $k$-systole comme minimum de ces fonctions longueurs :

$$
\operatorname{sys}_{k}=\inf \left\{l_{\left\{\gamma_{1}, \ldots, \gamma_{k}\right\}} ; \text { les } \gamma_{1}, \ldots, \gamma_{k} \text { sont } k \text { géodésiques f. s. disjointes }\right\} \text {. }
$$

Le cadre théorique développé pour la systole englobe ces nouveaux invariants. La constante de Bers apparaît naturellement en tant que borne supérieure de la $3 g-3$ systole.

\subsection{Plan de la démonstration}

Donnons une explication heuristique de la démonstration. Imaginons une éventuelle surface de Riemann $X$ réalisant la constante de Bers en genre 2. À l'instar des deux maxima connus, celle-ci posséderait probablement une géodésique séparante $\xi$ de longueur la 3-systole de $X$. Nous en déduirions (par certaines inégalités) que les trois points de Weierstrass dans chacun des tores bordés par $\xi$ seraient très proches les uns des autres, et inversement deux points de Weierstrass se situant chacun d'un côté de $\xi$ seraient très éloignés l'un de l'autre (Fig. 1). Ainsi, les autres triplets de géodésiques réalisant la 3 -systole de $X$, probablement formés de géodésiques non séparantes, vérifieraient la configuration suivante : deux géodésiques seraient petites et se situeraient chacune dans un tore bordé par $\xi$, tandis que la troisième relierait un point de Weierstrass de chaque tore, coupant $\xi$ en deux points. Pour les surfaces proches de $X$, la 3 -systole et la systole de la famille formée de $\xi$ et des géodésiques coupant $\xi$ en deux points coïncideraient donc; et nous serions ramenés à la recherche de surfaces maximales pour cette nouvelle fonction systole facile à appréhender.

La démonstration repose sur l'introduction d'un nouvel outil, le graphe de contiguïté, rendant compte de la répartition des points de Weierstrass sur une surface de Riemann hyperelliptique. Lorque la 3-systole est grande, cet outil nous informe que les points de Weierstrass se répartissent en deux groupes de trois points proches les uns des autres (parties 1 et 3). Il s'agit ensuite de montrer que la 3-systole est égale à la systole d'une famille de géodésiques reliant les deux groupes de points de Weierstrass (partie 4). Il reste alors à déterminer le maximum de cette fonction systole et à conclure (partie 5), ceci conduit naturellement à la construction d'un nouveau maximum local de la 3-systole. La partie 2 établit des résultats topologiques préliminaires. 
À partir de maintenant, $X$ désigne une surface de Riemann de genre 2. Sauf mention du contraire, par géodésique nous entendrons géodésique fermée simple incontractile. Lorsque cela ne prêtera pas à confusion, le nom d'une géodésique désignera encore sa longueur : nous préférerons $\cosh (\gamma)$ à $\cosh (l(\gamma))$.

\section{Graphe de contiguïté}

Parallèlement à P. Schmutz Schaller et ses techniques de découpages, C. Bavard développa dans [1] une approche de la systole des surfaces de Riemann reposant sur un argument de densité. Rappelons brièvement cet argument : soit $S$ une surface de Riemann hyperelliptique de genre $g \geq 2$, on considère dans le revêtement universel les cellules de Dirichlet-Voronoï associées aux relevés des points de Weierstrass, chacune d'elles contient un disque de centre le relevé d'un point de Weierstrass et de rayon la moitié du rayon d'injectivité ; on compare alors la densité des disques dans leurs cellules avec la densité maximale d'empilement—donnée par K. Böröczky dans [7]— afin d'obtenir un résultat, par exemple une borne sur la systole. En appliquant cette méthode en d'autres points que les points de Weierstrass, C. Bavard obtint également dans [2] une borne optimale sur le rayon maximal d'un disque plongé dans une surface de Riemann de genre fixé :

Théorème (Bavard) Soit $S$ une surface de Riemann de genre $g \geq 2$, s'il existe un disque ouvert de rayon $R$ plongé dans $S$ alors $\cosh R \leq \frac{1}{2 \sin \left(\frac{\pi}{12 g-6}\right)}$.

La méthode de Bavard conduit à deux observations intéressantes : premièrement, les points de Weierstrass jouent un rôle prépondérant et il serait utile d'étudier leur répartition sur la surface; deuxièmement, si une surface de Riemann de genre 2 possède trois géodésiques disjointes réalisant chacune le rayon d'injectivité en un point, alors sa 3-systole est petite. En effet, la borne sur le rayon d'injectivité de [2] permet de majorer la longueur de ces géodésiques par $2 \operatorname{arccosh}(2,88)$, or nous savons par [15] que $B(2)>2 \operatorname{arccosh}(4,67)$.

Suivant la première observation, nous allons introduire un graphe géodésique dont les sommets sont les points de Weierstrass. Nous tenterons alors de déterminer, à l'aide de la deuxième observation, les configurations possibles de ce graphe sous l'hypothèse de minoration : $\operatorname{sys}_{3} \geq 2 \operatorname{arccosh}(4,67)$.

\subsection{Définitions}

Nous allons provisoirement considérer une surface hyperbolique compacte $S$ munie d'une involution hyperelliptique $\iota_{S}$.

Définition 1.1 Nous appellerons graphe de contiguïté de la surface $S$, noté $G_{c o n t}(S)$, le graphe métrique obtenu en quotientant par l'involution hyperelliptique l'ensemble des géodésiques réalisant la distance entre un point de Weierstrass et l'ensemble des points de Weierstrass restants. 
Remarque 1.1 Le graphe de contiguïté d'une surfaces de Riemann se présente comme un graphe géodésique sur la sphère munie de la métrique à singularités coniques induite par l'involution hyperelliptique.

Exemple 1.1 Le graphe de contiguïté d'un tore à un bord est formé de trois sommets, et de deux ou trois arêtes. Génériquement il en possède deux, dont une provient de la systole. Lorsqu'il en possède trois, les deux arêtes les plus longues ont nécessairement même mesure.

Exemple 1.2 Le graphe de contiguïté de la surface de Bolza est le 1-squelette d'un octaèdre régulier [1].

La proposition suivante montre que les sommets du graphe de contiguïté sont exactement les points de Weierstrass.

Lemme 1.1 Soient $\gamma$ et $\gamma^{\prime}$ deux géodésiques distinctes réalisant chacune la distance entre un point de Weierstrass et l'ensemble des points de Weiestrass restants. Alors, les points d'intersection éventuels de $\gamma$ et $\gamma^{\prime}$ sont des points de Weierstrass.

Preuve Si $\gamma$ et $\gamma^{\prime}$ ont un point de Weierstrass en commun, l'assertion est évidente. Plaçons nous désormais dans le cas où les deux géodésiques ne passent pas par un même point de Weierstrass. Pour fixer les idées, disons que $\gamma$ passe par $w_{1}, w_{2}$ et réalise la distance entre $w_{1}$ et les autres points de Weierstrass, et que $\gamma^{\prime}$ passe par $w_{3}$, $w_{4}$ et réalise la distance entre $w_{3}$ et les autres points de Weierstrass. Supposons, par l'absurde, l'existence d'un point $p$ appartenant à $\gamma$ et $\gamma^{\prime}$. Si par exemple $d\left(p, w_{2}\right) \leq$ $d\left(p, w_{4}\right)$ alors on voit facilement que $d\left(w_{3}, w_{2}\right)<d\left(w_{3}, w_{4}\right)$, ce qui est bien absurde. De même, on trouvera une absurdité si $d\left(p, w_{2}\right) \geq d\left(p, w_{4}\right)$.

Le graphe de contiguïté est un objet trop complexe pour permettre une étude directe, c'est pourquoi nous allons nous concentrer sur une famille de sous-graphes.

Définition 1.2 Un graphe sera dit minimal s'il minimise le nombre d'arêtes parmi les graphes ayant même nombre de sommets et de composantes connexes.

Un sous-graphe d'un graphe $G$ sera qualifié de minimal s'il minimise le nombre d'arêtes parmi les sous-graphes de $G$ ayant même nombre de sommets et de composantes connexes que $G$.

Remarque 1.2 En termes de théorie des graphes, un graphe minimal est une forêt, et un sous-graphe minimal d'un graphe $G$ est une forêt maximale de $G$ (voir [10]).

Exemple 1.3 Nous avons représenté en Fig. 2 tous les graphes minimaux à six sommets sans sommet isolé, classés suivant leur nombre de composantes connexes.

Les graphes minimaux vont en quelque sorte éliminer l'information inutile contenue dans le graphe de contiguïté. De plus, ce sont des graphes au sens complexe simplicial fini de dimension 1, tandis que le graphe de contiguïté est un graphe au sens complexe cellulaire fini de dimension 1. 


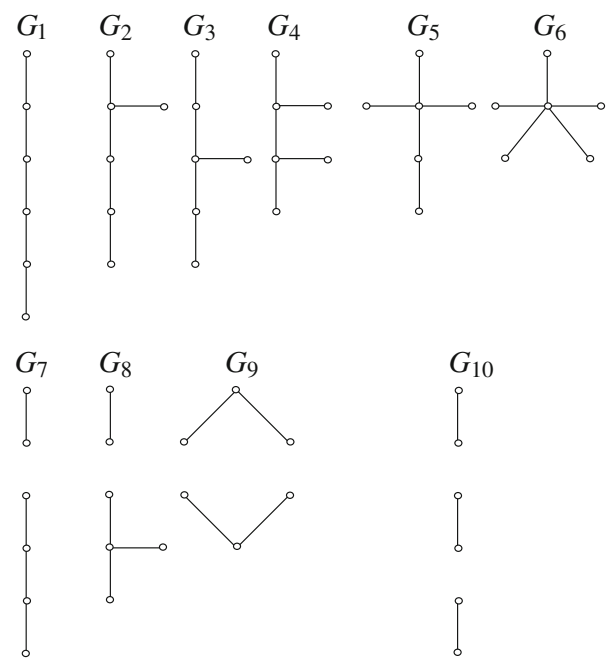

Fig. 2 Graphes minimaux à 6 sommets

1.2 Graphe de contiguïté en genre 2 sous hypothèse de minoration de la 3-systole

Dans ce paragraphe nous nous intéressons uniquement aux surfaces de Riemann de genre 2, et les énoncés ne sont valables que pour ces surfaces. La proposition suivante donne la configuration des sous-graphes minimaux du graphe de contiguiité sous une hypothèse de minoration de la 3-systole.

Proposition 1.1 Soit X une surface de Riemann de genre 2 vérifiant l'hypothèse $\operatorname{sys}_{3} \geq 2 \operatorname{arccosh}(4,67)$. Soit $G$ un graphe géodésique sur la sphère $X /\left\langle\iota_{X}\right\rangle$, ayant pour sommets les points coniques. Si aucun sommet n'est isolé, et si les arêtes sont de longueur majorée par arccosh $(2,88)$, alors tout sous-graphe minimal de G est isomorphe à $G_{9}$.

Corollaire 1.1 Sous l'hypothèse sys $_{3} \geq 2 \operatorname{arccosh}(4,67)$, tout sous-graphe minimal du graphe de contiguïté d'une surface de Riemann de genre 2 est isomorphe au graphe $G_{9}$.

Le corollaire 1.1 est une conséquence immédiate de la proposition ci-dessous, qui fera l'objet de la partie 3 de l'article.

Proposition 3.1 Sous l'hypothèse de minoration $\mathrm{sys}_{3} \geq 2 \operatorname{arccosh}(4,67)$, les arêtes du graphe de contiguïté d'une surface de Riemann de genre 2 sont de longueur inférieure à $\operatorname{arccosh}(2,88)$

Soit $X$ une surface de Riemann de genre 2 vérifiant $\operatorname{sys}_{3} \geq 2 \operatorname{arccosh}(4,67)$. Le quotient $X /\left\langle\iota_{X}\right\rangle$ est une sphère munie d'une métrique hyperbolique avec six singularités coniques d'angle $\pi$. Soit $G$ un graphe géodésique sur $X /\left\langle\iota_{X}\right\rangle$ tel que : les sommets de $G$ sont les points coniques, aucun sommet n'est isolé, la longueur des arêtes est majorée par $\operatorname{arccosh}(2.88)$. Alors $G$ ne possède pas trois arêtes disjointes, 


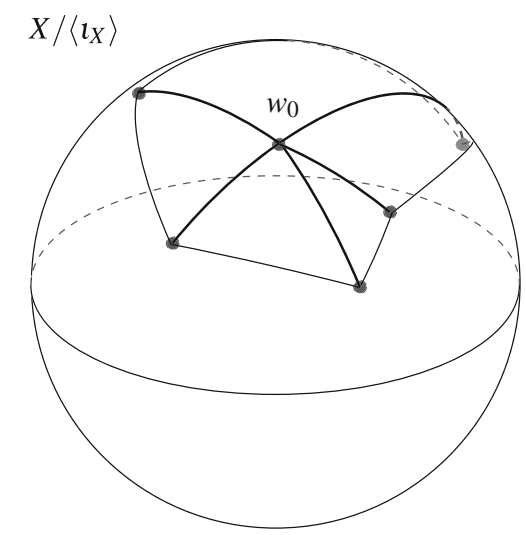

Fig. 3 Pentagone sur une sphère singulière

sinon les relevés de ces arêtes formeraient une partition de $X$ de longueur inférieure à $2 \operatorname{arccosh}(4,67)$. Dans le langage de la théorie des graphes on dit que $G$ n'a pas d'appariemment parfait (de perfect matching en anglais).

La preuve de la proposition 1.1 se divise en deux parties. Dans la première partie, nous éliminons les sous-graphes minimaux non isomorphes à $G_{4}$ et $G_{9}$ grâce à l'alternative suivante : ou bien les degrés des sommets sont petits, et il existe trois arêtes disjointes; ou bien le degré d'un sommet est grand, et il existe des voisins de ce sommet proches les uns des autres, ce qui permet de construire des arêtes petites disjointes. Dans la deuxième partie, nous éliminons les sous-graphes minimaux isomorphes à $G_{4}$. L'idée consiste à considérer la plus petite géodésique $\gamma$ disjointe de la géodésique reliant les deux points de Weierstrass correspondant aux sommets de degré 3.

Preuve de la proposition 1.1 Plaçons-nous dans la situation de l'énoncé. Nous venons de voir que le graphe $G$ ne possède pas trois arêtes disjointes, il s'ensuit qu'aucun sous-graphe de $G$ n'est isomorphe à $G_{1}, G_{3}, G_{7}$ ou $G_{10}$. Nous allons maintenant montrer qu' aucun sous-graphe de $G$ n'est isomorphe à $G_{4}, G_{6}$ ou $G_{8}$; comme $G_{8}$ est un sous-graphe de $G_{2}$ et $G_{5}$, cela suffira pour conclure.

Supposons que $G$ contient un sous-graphe $H$ isomorphe à $G_{6}$. Appelons $w_{0}$ le sommet de degré 5 , et $w_{i} i=1 \ldots 5$ ses voisins que nous supposerons ordonnés suivant une orientation de la sphère. Ajoutons à $H$ les segments géodésiques complétant les paires d'arêtes $\left(w_{0} w_{i}, w_{0} w_{i+1}\right)$ (avec la convention évidente $\left.w_{6}=w_{1}\right)$ en des triangles hyperboliques sans singularité. Nous avons ainsi construit un pentagone hyperbolique, pas nécessairement convexe, avec une singularité conique (Fig. 3). L'angle total en $w_{0}$ étant $\pi$, il existe trois angles $w_{i} \widehat{w_{0} w_{i}}$ de mesures inférieures ou égales à $\pi / 3$. Comme les arêtes $w_{0} w_{i}$ sont de longueur inférieure à $\operatorname{arccosh}(2,88)$, les côtés du pentagone faisant face aux angles plus petits que $\pi / 3$ sont de longueur inférieure à $\operatorname{arccosh}(4,67)$. Parmi ces côtés, nous pouvons en choisir deux disjoints, et leur ajouter l'arête $w_{0} w_{i}$ reliant les deux sommets non encore utilisés. Ces trois segments se relèvent en une partition de $X$ de longueur inférieure à $2 \operatorname{arccosh}(4.67)$, ce qui est absurde.

Supposons que $G$ contient un sous-graphe isomorphe à $G_{8}$. Appelons $w_{0}$ le sommet de degré $3, w_{1}, w_{2}, w_{3}$ ses voisins, et $w_{4}, w_{5}$ les sommets restants. Comme 


\section{$X$}

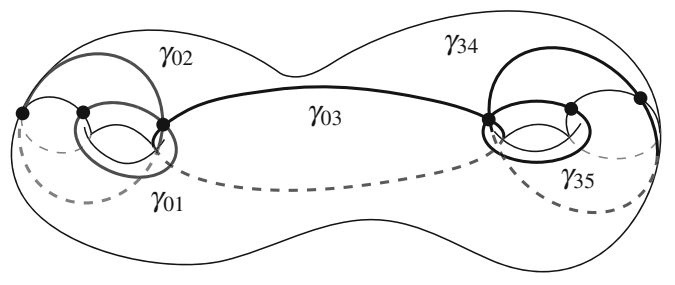

$H$

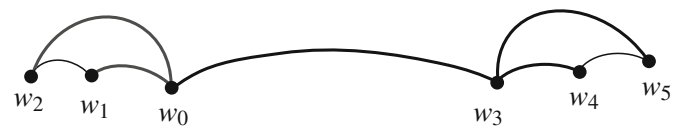

Fig. 4 Sous-graphe isomorphe à $G_{4}$

précédemment, on construit un polygone hyperbolique singulier ayant pour sommets les voisins de $w_{0}$. Ce polygone est disjoint de l'arête $w_{4} w_{5}$. L'un des côtés de ce polygone, disons $w_{2} w_{3}$, est de longueur inférieure à $\operatorname{arccosh}(4,67)$. Ainsi les géodésiques au-dessus du côté $w_{2} w_{3}$ et des arêtes $w_{0} w_{1}, w_{4} w_{5}$ forment une partition de longueur inférieure à $2 \operatorname{arccosh}(4,67)$, ce qui est absurde.

Supposons que $G$ contient un sous-graphe $H$ isomorphe à $G_{4}$. Nous adopterons les notations de la Fig. 4, en particulier $w_{0}$ et $w_{3}$ seront les sommets de degré 3 . Il est impossible d'ajouter à $H$ une arête de longueur inférieure à $\operatorname{arccosh}(4,67)$ reliant $w_{1}$ à $w_{2}$ (resp. $w_{4}$ à $w_{5}$ ), sinon en appliquant le raisonnement du paragraphe précédent au graphe formé des arêtes $w_{1} w_{2}, w_{0} w_{3}, w_{3} w_{4}, w_{3} w_{5}$ (resp. $w_{4} w_{5}, w_{0} w_{3}, w_{0} w_{1}$, $w_{0} w_{2}$ ) nous trouverions une absurdité. De même, il est impossible d'ajouter à $H$ une arête de longueur inférieure à $\operatorname{arccosh}(2,88)$ entre deux sommets $w_{i}, w_{j}$ tels que $\left\{w_{i}, w_{j}\right\} \neq\left\{w_{0}, w_{3}\right\}$, sinon nous serions ramenés à un des cas traités précédemment.

Travaillons directement dans la surface $X$, nous noterons $\gamma_{i j}$ la géodésique de $X$ correspondant à l'arête $w_{i} w_{j}$. Comme les géodésiques $\gamma_{01}$ et $\gamma_{02}$ (resp. $\gamma_{34}$ et $\gamma_{35}$ ) s'intersectent en exactement un point, il existe un unique tore à un bord $\mathbb{T}_{1}$ (resp. $\left.\mathbb{T}_{2}\right)$ les contenant. Dans ce tore, il existe deux géodésiques complétant $\gamma_{01}$ et $\gamma_{02}$ (resp. $\gamma_{34}$ et $\gamma_{35}$,) en des triangles, mais seulement l'une d'elles n'intersecte pas $\gamma_{03}$. Nous appellerons $\gamma_{12}$ (resp. $\gamma_{45}$ ) cette géodésique. D'après le paragraphe précédent $\gamma_{12}$ et $\gamma_{45}$ sont en longueur supérieures à $2 \operatorname{arccosh}(4,67)$. On en déduit, que dans le triangle $\left(w_{0} w_{1} w_{2}\right)\left(\right.$ resp. $\left.\left(w_{3} w_{4} w_{5}\right)\right)$ les points $w_{1}$ et $w_{2}$ (resp. $w_{4}$ et $w_{5}$ ) sont à une distance supérieure à $\operatorname{arccosh}(1,62)$ de leur côté opposé (simple utilisation du théorème de Pythagore hyperbolique).

Soit $\gamma$ la plus petite géodésique disjointe de $\gamma_{03}$. Nous allons établir une liste des propriétés de $\gamma$. Tout d'abord, $\gamma$ est de longueur inférieure à $2 \operatorname{arccosh}(2,88)$ par l'inégalité systolique du tore à deux bords [15], $\S 5$. Ensuite, $\gamma$ n'est pas séparante, sinon nous pourrions construire une petite partition en appliquant l'inégalité systolique du tore à un bord $[15,13,11]$. En particulier $\gamma$ passe par deux points de Weierstrass différents de $w_{0}$ et $w_{3}$. Enfin, $\gamma$ n'est contenue dans aucun des tores $\mathbb{T}_{1}, \mathbb{T}_{2}$, sinon nous aurions $\gamma=\gamma_{12}$ ou $\gamma=\gamma_{45}$ car $\gamma$ n'intersecte pas $\gamma_{03}$, mais ceci est impossible vu les longueurs de ces géodésiques. Pour finir, remarquons que $\gamma$ intersecte une 
des géodésiques $\gamma_{01}, \gamma_{02}, \gamma_{34}, \gamma_{35}$ en dehors des points de Weierstrass. Dans le cas contraire, en ajoutant l'image de $\gamma$ dans $X /\left\langle\iota_{X}\right\rangle$ au graphe $H$, nous ajouterions une nouvelle arête de longueur inférieure à $\operatorname{arccosh}(2,88)$ entre deux sommets différents de $w_{0}$ et $w_{3}$, or nous avons vu plus haut que c'est impossible.

Pour fixer les idées, nous supposerons que $\gamma$ passe par $w_{1}$. Partant de $w_{1}$, la géodésique $\gamma$ va quitter le tore $\mathbb{T}_{1}$. Pour des raisons de minimalité, les géodésiques $\gamma_{01}$ et $\gamma$ ne s'intersectent pas en dehors de $w_{1}$. Donc, avant de quitter $\mathbb{T}_{1}, \gamma$ peut intersecter au plus une fois $\gamma_{02}$. Dans ce cas, la distance parcourue par $\gamma$ avant de quitter $\mathbb{T}_{1}$ est supérieure à $\operatorname{arccosh}(1,62)$, comme vu plus haut. Le même raisonnement vaut pour le deuxième point de Weierstrass appartenant à $\gamma$ : partant du deuxième point de Weierstrass, $\gamma$ peut intersecter au plus une géodésique $\gamma_{i j}$ avant de changer de tore, et dans ce cas elle parcourt une distance supérieure à $\operatorname{arccosh}(1,62)$ avant d'atteindre le bord du tore. Cependant $\gamma / 2<\operatorname{arccosh}(2,88)<2 \operatorname{arccosh}(1,62)$, donc le comportement décrit ci-dessus ne se produit que pour au plus un point de Weierstrass. De ce fait, nous supposerons que $\gamma$ part de $w_{1}$ et quitte le tore $\mathbb{T}_{1}$ sans intersecter ni $\gamma_{01}$ ni $\gamma_{02}$. Après avoir quitter $\mathbb{T}_{1}$ elle intersecte nécessairement $\gamma_{34}$ ou $\gamma_{35}$, disons $\gamma_{34}$.

Comme $\gamma$ et $\gamma_{34}$ sont de longueur inférieure à $2 \operatorname{arccosh}(2,88)$, on construit à partir de ces deux géodésiques un chemin géodésique par morceaux reliant $w_{1}$ à $w_{3}$ ou $w_{4}$, de longueur inférieure à $\operatorname{arccosh}(2,88)$, et n'intersectant aucune $\gamma_{i j}$ en dehors des points de Weierstrass. Ce chemin, vu dans la sphère $X /\left\langle\iota_{X}\right\rangle$, correspond à une nouvelle arête du graphe $H$, cette arête fait apparaître un sous-graphe isomorphe à $G_{1}$ ou $G_{8}$, ce qui est impossible.

\section{Géométrie-Topologie associée à une géodésique séparante}

Soit $X$ une surface de Riemann de genre 2. Nous nous intéressons ici aux relations entre les géodésiques séparantes et les géodésiques non séparantes de $X$. En particulier, étant fixée une géodésique séparante $\xi$, on étudie la famille $\mathcal{G}_{\xi}$ des géodésiques intersectant $\xi$ en exactement deux points. Ce travail est motivé par le fait qu'à chaque géodésique $\gamma$ de cette famille, on associe naturellement la partition formée de $\gamma$ et des deux géodésiques disjointes de $\xi$ et $\gamma$. Rappelons que par géodésique nous entendons une géodésique fermée simple incontractile.

\subsection{Géodésique séparante déterminée par deux non séparantes}

Notons $\mathcal{G}_{s}$ (resp. $\mathcal{G}_{\S}$ ) l'ensemble des géodésiques fermées simples séparantes (resp. non séparantes) de $X$. Considérons la partie $\mathcal{A}$ de $\mathcal{G}_{\forall} \times \mathcal{G}_{\mathscr{y}}$ formée des couples de géodésiques non séparantes s'intersectant en exactement un point :

$$
\mathcal{A}=\left\{(\alpha, \beta) \in \mathcal{G}_{\mathscr{S}} \times \mathcal{G}_{\mathscr{A}} ; \alpha \text { et } \beta \text { s'intersectent en exactement un point }\right\} .
$$

Nous pouvons construire une application surjective

$$
\begin{aligned}
& \text { Sép : } \mathcal{A} \subset \mathcal{G}_{\mathscr{g}} \times \mathcal{G}_{\mathscr{g}} \longrightarrow \mathcal{G}_{s} \\
& (\alpha, \beta) \longmapsto \operatorname{Sép}(\alpha, \beta),
\end{aligned}
$$


en associant à chaque couple $(\alpha, \beta) \in \mathcal{A}$ l'unique géodésique fermée simple dans la classe d'homotopie libre du commutateur $[\alpha, \beta]$. Le point d'intersection des deux géodésiques a implicitement été choisi comme point base du groupe fondamental. L'application Sép ainsi définie est bien à valeurs dans l'ensemble des géodésiques séparantes, puisque la classe du commutateur dans $H_{1}(X, \mathbf{Z})$ est nulle.

Proposition 2.1 Soit $(\alpha, \beta) \in \mathcal{A}$, alors une géodésique fermée simple n'intersectant pas $\alpha \cup \beta$ est contenue dans le tore bordé par $\operatorname{Sép}(\alpha, \beta)$ ne contenant ni $\alpha$ ni $\beta$.

Preuve Soit $\gamma$ une géodésique fermée simple de $X$ disjointe de $\alpha \cup \beta$, $\gamma$ n'a pas d'homotopie dans le tore bordé par $\operatorname{Sép}(\alpha, \beta)$ contenant $\alpha$ et $\beta$, autrement dit la trace de $\gamma$ sur ce tore est homotope à un arc du bord. Ainsi, $\gamma$ est homotope à une courbe n'intersectant pas $\operatorname{Sép}(\alpha, \beta)$. Comme les géodésiques minimisent le nombre d'intersection nous concluons directement.

Remarque 2.1 Une géodésique disjointe de $\alpha$ et $\beta$ est aussi disjointe de $\operatorname{Sép}(\alpha, \beta)$. En particulier, $\operatorname{Sép}(\alpha, \beta)$ est l'unique géodésique séparante n'intersectant pas $\alpha \cup \beta$.

\subsection{Rayons des tores à un bord}

Soit $\mathbb{T}$ un tore à un bord hyperbolique. Nous appellerons rayon de $\mathbb{T}$ un segment géodésique simple reliant deux points du bord, orthogonal au bord en ces deux points, et stable par l'involution hyperelliptique. Étant donné une géodésique fermée simple $\gamma$ de $\mathbb{T}$, il existe un unique rayon $\gamma^{\prime}$ de $\mathbb{T}$ disjoint de $\gamma$. En fait, cette relation induit une bijection entre les géodésiques fermées simples et les rayons de $\mathbb{T}$. On désignera $\gamma^{\prime}$ comme le rayon dual de $\gamma$. Les longueurs de $\gamma$ et $\gamma^{\prime}$ sont liées par la formule $\sinh \left(\gamma^{\prime} / 2\right) \sinh \left(l_{\partial}(\mathbb{T}) / 4\right)=\cosh (\gamma / 2)$ avec $l_{\partial}(\mathbb{T})$ la longueur du bord de $\mathbb{T}$. Soulignons qu'un rayon de $\mathbb{T}$ minimise la longueur dans sa classe d'homotopie libre. Nous renvoyons à [11] pour plus de détails.

\subsection{Correspondance induite par une géodésique séparante}

Fixons-nous $\xi$ une géodésique fermée simple séparante de $X$, nous souhaitons comprendre la géométrie-topologie associée au couple $(X, \xi)$.

Notations Nous noterons $\mathbb{T}_{1}$ et $\mathbb{T}_{2}$ les tores à un bord issus de la découpe de $\xi$ dans $X$. Par $\mathcal{G}_{\mathbb{T}_{i}}$ nous désignerons l'ensemble des géodésiques fermées simples de $\mathbb{T}_{i}$, et par $\mathcal{G}_{\xi}$ l'ensemble des géodésiques fermées simples de $X$ coupant $\xi$ en exactement deux points.

Les géodésiques fermées simples de $X$ étant stables par l'involution hyperelliptique, chaque géodésique $\gamma \in \mathcal{G}_{\xi}$ passe nécessairement par un point de Weierstrass de chacun des tores $\mathbb{T}_{1}, \mathbb{T}_{2}$. Bien sûr, il existe des géodésiques fermées simples coupant $\xi$ en plus de deux points, nous pouvons même les choisir passant par deux points de Weierstrass situés d'un même côté de $\xi$.

Nous allons mettre en correspondance $\mathcal{G}_{\xi}$ avec $\mathcal{G}_{\mathbb{T}_{1}} \times \mathcal{G}_{\mathbb{T}_{2}}$ via le lemme suivant : 


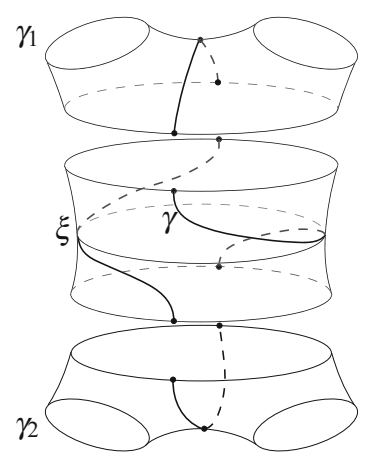

Fig. 5 Décomposition d'un élément de $\mathcal{G}_{\xi}$

Lemme 2.1 Soit $\gamma \in \mathcal{G}_{\xi}$, il existe un unique couple $\left(\gamma_{1}, \gamma_{2}\right) \in \mathcal{G}_{\mathbb{T}_{1}} \times \mathcal{G}_{\mathbb{T}_{2}}$ tel que $\gamma_{1}$ et $\gamma_{2}$ soient disjointes de $\gamma$.

Preuve Cette propriété est purement topologique. En découpant la trace de $\gamma$ sur le tore $\mathbb{T}_{i}$ nous obtenons un cylindre. À homotopie et orientation près, il existe une unique courbe fermée simple sur ce cylindre, $\gamma_{i}$ est la géodésique dans cette classe d'homotopie libre.

L'appplication $\Xi_{\xi}$ ci-dessous est donc bien définie,

$$
\begin{aligned}
\Xi_{\xi}: \mathcal{G}_{\xi} & \longrightarrow \mathcal{G}_{\mathbb{T}_{1}} \times \mathcal{G}_{\mathbb{T}_{2}} \\
\gamma & \longmapsto\left(\gamma_{1}, \gamma_{2}\right),
\end{aligned}
$$

elle est par ailleurs équivariante pour l' action naturelle du groupe $\left\langle t_{\xi / 2}\right\rangle$, où $t_{\xi / 2}$ désigne le demi-twist selon $\xi$. Lorsqu'il n'y aura pas d'ambiguïté nous omettrons l'indice $\xi$ de $\Xi_{\xi}$.

Lemme 2.2 Soient $\gamma$ et $\delta$ deux éléments de $\mathcal{G}_{\xi}$, nous avons l'équivalence

$$
\Xi(\gamma)=\Xi(\delta) \Longleftrightarrow \exists k \in \mathbf{Z}, \delta=\left(t_{\xi / 2}\right)^{k} \cdot \gamma
$$

Preuve L'implication réciproque est triviale car $\xi$ et $\gamma$ n'intersectent pas $\gamma_{1}$ et $\gamma_{2}$. Soit $\left(\gamma_{1}, \gamma_{2}\right) \in \mathcal{G}_{\mathbb{T}_{1}} \times \mathcal{G}_{\mathbb{T}_{2}}$, déterminons tous les antécédents de ce couple par $\Xi$. Découpons $\gamma_{1}$ et $\gamma_{2}$ dans $X$, nous voyons sans difficulté qu'un antécédent $\gamma$ intersecte seulement une fois la perpendiculaire commune aux deux bords issus de la découpe de $\gamma_{i}(i=1$ ou 2$)$. Nous pouvons de ce fait visualiser les antécédents de $\left(\gamma_{1}, \gamma_{2}\right)$ de la façon suivante (Fig. 5) : nous divisons $X$ en trois parties, deux pantalons et un cylindre, en découpant deux courbes homotopes à $\xi$. Fixons deux points sur chacune de ces courbes, un antécédent de $\left(\gamma_{1}, \gamma_{2}\right)$ est homotope à une courbe dont le tracé dans chacun des pantalons relie simplement les points que nous avons fixés en passant entre les deux autres bords. Dans le cylindre, la courbe possède deux composantes connexes, chaque composante relie un point fixé d'un bord à un point fixé de l'autre 


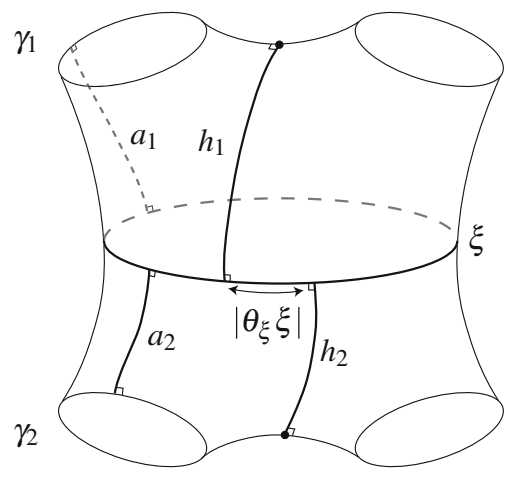

Fig. 6 Notations

bord. Il est clair que toutes ces courbes sont dans le même orbite sous l'action de $\left\langle t_{\xi / 2}\right\rangle$.

Le groupe $\left\langle t_{\xi / 2}\right\rangle$ agit librement sur $\mathcal{G}_{\xi}$ et trivialement sur $\mathcal{G}_{1}$ et $\mathcal{G}_{2}$. Gardons le même nom $\Xi$ pour l'application entre les quotients, nous avons :

Proposition 2.2 L'application $\Xi$ établit une bijection entre $\mathcal{G}_{\xi} /\left\langle t_{\xi / 2}\right\rangle$ et $\mathcal{G}_{\mathbb{T}_{1}} \times \mathcal{G}_{\mathbb{T}_{2}}$.

\subsection{Relation entre les longueurs}

Pour le problème qui nous intéresse, à savoir la 3-systole, la correspondance définie ci-dessus nous sera utile dans la mesure où elle induit une relation —une égalitéentre les longueurs des géodésiques.

Revenons au couple $(X, \xi)$, et fixons $\gamma \in \mathcal{G}_{\xi}$. Dans le pantalon issu de la découpe de $\gamma_{i}$ dans $\mathbb{T}_{i}$ notons : $a_{i}$ une perpendiculaire commune à $\xi$ et à un bord identifié à $\gamma_{i}, h_{i}$ un segment moitié de $\gamma_{i}^{\prime}$ le rayon dual de $\gamma_{i}$ dans $\mathbb{T}_{i}$. Enfin, désignons par $\theta_{\xi}$ la coordonnée de twist selon $\xi$ relativement à $h_{1}$ et $h_{2}$. Nous convenons qu'à $\theta_{\xi}=0$ la géodésique $\gamma$ supporte les segments $h_{1}, h_{2}$. Ces notations sont résumées par la Fig. 6 .

À twist quelconque, la longueur de $\gamma$ est donnée dans [8] par la formule :

$$
\begin{aligned}
\cosh (\gamma / 2)= & \sinh \left(\gamma_{1} / 2\right) \sinh \left(\gamma_{2} / 2\right)\left[\sinh \left(a_{1}\right) \sinh \left(a_{2}\right) \cosh \left(\theta_{\xi} \xi\right)+\cosh \left(a_{1}\right) \cosh \left(a_{2}\right)\right] \\
& -\cosh \left(\gamma_{1} / 2\right) \cosh \left(\gamma_{2} / 2\right) .
\end{aligned}
$$

Les égalités suivantes :

$$
\left\{\begin{array}{l}
\sinh \left(\gamma_{i} / 2\right) \sinh \left(a_{i}\right)=\cosh \left(h_{i}\right) \\
\sinh \left(\gamma_{i} / 2\right) \cosh \left(a_{i}\right)=\cosh \left(\gamma_{i} / 2\right) \operatorname{coth}(\xi / 4) \quad i=1,2 ; \\
\sinh \left(h_{i}\right) \sinh (\xi / 4)=\cosh \left(\gamma_{i} / 2\right)
\end{array}\right.
$$


nous permettent d'obtenir l'expression

$$
\begin{aligned}
\cosh (\gamma / 2)= & \cosh \left(h_{1}\right) \cosh \left(h_{2}\right) \cosh \left(\theta_{\xi} \xi\right) \\
& +\cosh \left(\gamma_{1} / 2\right) \cosh \left(\gamma_{2} / 2\right)\left[\operatorname{coth}^{2}(\xi / 4)-1\right], \\
\cosh (\gamma / 2)= & \cosh \left(h_{1}\right) \cosh \left(h_{2}\right) \cosh \left(\theta_{\xi} \xi\right)+\sinh \left(h_{1}\right) \sinh \left(h_{2}\right) .
\end{aligned}
$$

\section{Majoration de la longueur des arêtes du graphe de contiguiité}

Le but de cette partie est de démontrer la proposition suivante :

Proposition 3.1 Sous l'hypothèse de minoration $\mathrm{sys}_{3} \geq 2 \operatorname{arccosh}(4,67)$, les arêtes du graphe de contiguïté d'une surface de Riemann de genre 2 sont de longueur inférieure à $\operatorname{arccosh}(2,88)$.

Dans toute cette partie, $X$ est une surface de Riemann de genre 2, et $\xi$ une géodésique fermée simple séparante de $X$. Rappelons que, sauf mention du contraire, par géodésique nous entendons une géodésique fermée simple incontractile.

\subsection{Rayon d'injectivité et graphe de contiguïté}

Une arête du graphe de contiguïté est un segment réalisant la distance entre un point de Weierstrass et l'ensemble des points de Weierstrass restants. La borne de Bavard sur le rayon d'un disque plongé — dont on parla au début de la partie 1 - fut le résultat motivant l'introduction de ce graphe. Il semblait a prioi simple d'obtenir une borne sur la longueur des arêtes du graphe de contiguïté en l'utilisant. Cependant si $\lambda$ est un lacet géodésique réalisant le rayon d'injectivité en un point de Weierstrass, ce lacet n'est pas nécessairement une géodésique, en particulier il ne passe pas forcément par un deuxième point de Weierstrass. Ainsi, la géodésique fermée $\lambda \cdot \iota_{X}(\lambda)^{-1}$, formée de $\lambda$ et de l'inverse de son image par l'involution hyperelliptique, n'est pas en général simple. Il se peut que ce soit topologiquement un huit, avec pour point double le point de Weierstrass. Dans ce cas, nous n'avons plus de contrôle sur la longueur des arêtes du graphe de contiguité adjacentes à ce point de Weierstrass.

En fait, il n'existe pas de borne sur la longueur des arêtes du graphe de contiguïté uniforme sur l'espace de Teichmüller. On construit un contre-exemple de la façon suivante : soit $X$ une surface de Riemann de genre 2 , on considère une partition de $X$ formée d'une géodésique séparante $\xi$ et de deux géodésiques non séparantes $\alpha_{1}$ et $\alpha_{2}$; en faisant tendre les longueurs de $\xi, \alpha_{1}, \alpha_{2}$ vers 0 , les arêtes de $G_{c o n t}(X)$ adjacentes aux deux points de Weierstrass par lesquels ne passent pas $\alpha_{1}$ et $\alpha_{2}$ dépassent n'importe quelle longueur, en conséquence du lemme du collier. Ce contre-exemple se généralise en genre supérieur.

\subsection{Notations}

Notations Étant fixée $\xi$, nous noterons $\mathbb{T}_{1}$ et $\mathbb{T}_{2}$ les tores bordés par $\xi$, nous nommerons $\alpha_{i}$ une géodésique réalisant la systole de $\mathbb{T}_{i}$, et $\beta_{i}$ la plus courte géodésique 


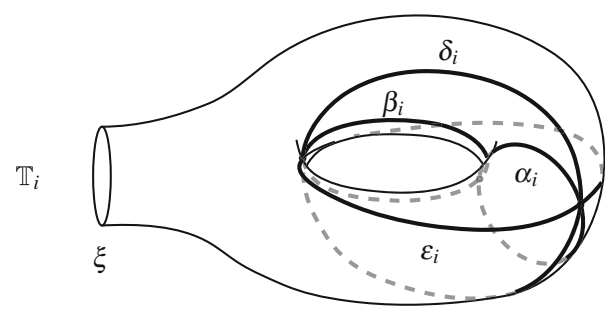

Fig. 7 Géodésiques dans le tore à un bord

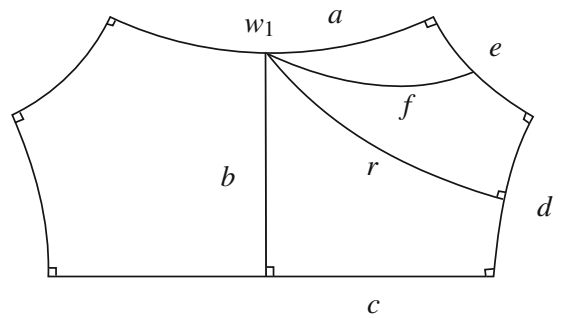

Fig. 8 Notations dans le pentagone droit

de $\mathbb{T}_{i}$ intersectant $\alpha_{i}$ en exactement un point. Enfin nous appellerons $\delta_{i}$ et $\varepsilon_{i}$ les deux géodésiques formant un triangle avec $\alpha_{i}$ et $\beta_{i}$ dans le tore $\mathbb{T}_{i}$, avec la convention $\delta_{i} \leq \varepsilon_{i}$ (voir Fig. 7).

Les géodésiques $\alpha_{i}, \beta_{i}, \delta_{i}, \varepsilon_{i}$ vérifient $\alpha_{i} \leq \beta_{i} \leq \delta_{i} \leq \varepsilon_{i}$, et par monotonie leurs rayons duaux vérifient eux aussi $\alpha_{i}^{\prime} \leq \beta_{i}^{\prime} \leq \delta_{i}^{\prime} \leq \varepsilon_{i}^{\prime}$ (se reporter au $\S 2.2$ ). Par définition, $\alpha_{i}$ est la plus courte géodésique de $\mathbb{T}_{i}$ et, par le lemme ci-dessous, $\beta_{i}$ est la deuxième plus courte géodésique de $\mathbb{T}_{i}$. En particulier $\alpha_{i}$ et $\beta_{i}$ se projettent sur deux arêtes de $G_{\text {cont }}\left(\mathbb{T}_{i}\right)$.

Lemme 3.1 La géodésique fermée simple de $\mathbb{T}_{i}$ la plus courte après $\alpha_{i}$ est $\beta_{i}$.

Remarque 3.1 Ce résultat est faux si l'on ne se restreint pas aux géodésiques fermées simples, c'est la discussion du paragraphe précédent. Par ailleurs le choix de $\beta_{i}$ n'est pas toujours unique.

Preuve Travaillons dans le tore $\mathbb{T}_{1}$, et notons $w_{1}$ le point de Weierstrass par lequel ne passe pas $\alpha_{1}$. La géodésique $\beta_{1}$ passe nécessairement par $w_{1}$, et se projette sur une arête de $G_{\text {cont }}\left(\mathbb{T}_{i}\right)$. Ainsi, $\beta_{1}$ est la géodésique la plus courte passant par $w_{1}$.

Soit $\eta$ une géodésique distincte de $\alpha_{1}$ ne passant pas par $w_{1}$. Nous allons montrer que $\eta \geq \beta_{1}$, ce qui suffit pour conclure. En découpant $\alpha_{1}$, puis les perpendiculaires communes aux bords issus de $\alpha_{1}$ et $\xi$, nous obtenons deux hexagones droits isométriques. L'un d'eux est représenté en Fig. 8 avec les conventions $e=\alpha_{1} / 2, c=\xi / 4$. Dans le pantalon, un segment moitié de $\eta$ relie les deux bords issus de la découpe de $\alpha_{1}$, et de ce fait $\eta / 2 \geq 2 a$. Le segment $f$, reliant $w_{1}$ au milieu de $e$, majore la demilongueur de la plus petite géodésique non triviale passant par $w_{1}$, d'où $f \geq \beta_{1} / 2 \geq e$. En appliquant l'inégalité triangulaire dans le triangle de côtés $a, f$ et $e / 2$ on a $a>e / 2$. Donc $\eta / 2 \geq 2 a>a+e / 2 \geq \beta_{1} / 2$. 


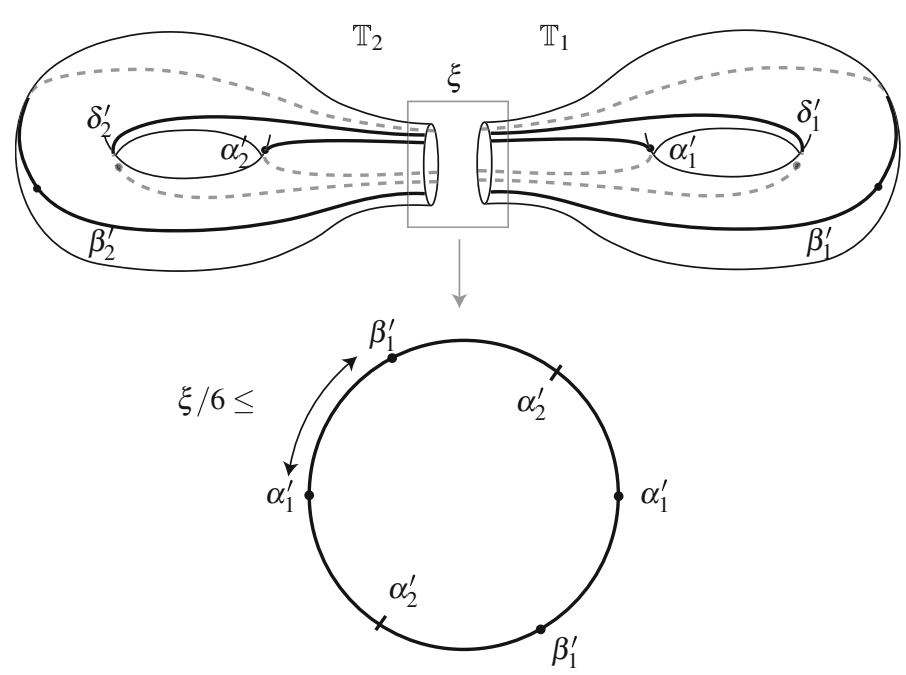

Fig. 9 Majoration du twist

\subsection{Majoration du twist}

Lemme 3.2 Il existe un point de $\xi \cap\left(\alpha_{1}^{\prime} \cup \beta_{1}^{\prime}\right)$ et un point de $\xi \cap \alpha_{2}^{\prime}$ séparés par un segment de $\xi$ de longueur inférieure à $\xi / 6$.

Preuve Les points de $\xi \cap\left(\alpha_{1}^{\prime} \cup \beta_{1}^{\prime}\right)$ divisent $\xi$ en quatre segments : deux contenant un point de $\delta_{1}^{\prime}$, et deux n'en contenant pas. Soit $Y_{1}$ la surface hyperbolique fermée de caractéristique -1 associée à $\mathbb{T}_{1}$ par autorecollement de $\xi=\partial \mathbb{T}_{1}$ (voir [11]). La découpe des rayons $\alpha_{1}^{\prime}$, $\beta_{1}^{\prime}$ et $\delta_{1}^{\prime}$ transforme la surface $Y_{1}$ en un pantalon hyperbolique. Les perpendiculaires communes de ce pantalon s'identifient à l'image de $\xi$ dans $Y_{1}$, et la perpendiculaire commune la plus longue est celle opposée au bord le plus long, celui provenant de $\delta_{1}^{\prime}$. La longueur de ce segment, qui est un de ceux ne contenant pas de point de $\delta_{1}^{\prime}$ est donc supérieure à $\xi / 6$.

Découpons $\alpha_{1}$ dans $\mathbb{T}_{1}$, nous avons un pantalon se divisant en deux hexagones droits dans lesquels nous situons sans difficultés les rayons $\alpha_{1}^{\prime}$ et $\beta_{1}^{\prime}$. Nous majorons directement la longueur d'un segment de $\xi$ ne contenant pas de point de $\delta_{1}^{\prime}$ par $\xi / 4$.

Comme la somme des longueurs des quatre segments délimités par les points de $\xi \cap\left(\alpha_{1}^{\prime} \cup \beta_{1}^{\prime}\right)$ vaut $\xi$, il est clair que les segments les plus courts sont ceux ne contenant pas de points de $\delta_{1}^{\prime}$, en particulier ces segments réalisent la distance sur $\xi$ entre $\alpha_{1}^{\prime}$ et $\beta_{1}^{\prime}$. On conclut en considérant un segment moitié de $\xi$ délimités par les extrémités du rayon $\alpha_{2}^{\prime}$, on a représenté la situation en Fig. 9, le résultat est maintenant évident.

Remarque 3.2 Au cours de la preuve, on a montré que la distance sur $\xi$ entre $\alpha_{1}^{\prime}$ et $\beta_{1}^{\prime}$ est comprise entre $\xi / 6$ et $\xi / 4$. Notons aussi que le résultat est optimal.

\subsection{Trois majorations connues}

Soient $X$ une surface de Riemann de genre 2, et $\xi$ une géodésique séparante de $X$. Alors 


$$
\begin{aligned}
& \cosh \left(R_{w_{j}}\right)<2,88, \\
& \sinh \left(\alpha_{i}^{\prime} / 2\right) \leq \frac{1}{2 \sinh (\xi / 12)}, \\
& \cosh \left(\alpha_{i} / 2\right) \leq \cosh (\xi / 6)+1 / 2,
\end{aligned}
$$

où $R_{w_{j}}$ désigne le rayon d'injectivité au point de Weierstrass $w_{j}, \alpha_{i}^{\prime}$ le plus petit rayon du tore $\mathbb{T}_{i}, \alpha_{i}$ la systole du tore $\mathbb{T}_{i}$. La première inégalité vient de la borne de Bavard sur le rayon d'un disque plongé, la deuxième est l'inégalité optimale pour le rayon d'un collier [4], la troisième est l'inégalité systolique optimale du tore à un bord $[15,13,11]$.

Conséquence 3.5 De (3.3) nous déduisons que si $\operatorname{sys}_{3}(X) \geq 2 \operatorname{arccosh}(4,67)$, alors toute géodésique séparante $\xi$ de $X$ vérifie

$$
\cosh (\xi / 2) \geq 4,67 \text {. }
$$

\subsection{Preuve de la proposition}

Considérons $X$ une surface de Riemann de genre 2 vérifiant $\operatorname{sys}_{3} \geq 2 \operatorname{arccosh}(4,67)$. Nous supposerons qu'en un point de Weierstrass $w_{1}$ de $X$ le rayon d'injectivité n'est pas réalisé par une géodésique fermée simple, mais par un lacet géodésique simple $\lambda$. Dans le cas contraire, on a une majoration de la longueur des arêtes de $G_{\text {cont }}(X)$ par (3.1).

\subsubsection{Lacet réalisant le rayon d'injectivité}

Lemme 3.3 Le lacet $\lambda$ n'est pas séparant.

Preuve La géodésique dans la classe d'homotopie libre de $\lambda$ est de longueur inférieure à $\lambda$, en particulier elle ne vérifie pas (3.4).

Avec le lacet $\lambda$, on considère naturellement la géodésique fermée simple séparante $\xi$ dans la classe d'homotopie libre du produit $\lambda \cdot \iota_{X}(\lambda)$, où $\iota_{X}(\lambda)$ est le lacet image de $\lambda$ par l'involution hyperelliptique. Cette géodésique est visiblement simple. Elle est séparante car $\left[\iota_{X}(\lambda)\right]=-[\lambda]$ dans $H_{1}(X, \mathbf{Z})$ et $\left[\iota_{X}(\lambda)\right] \neq[\lambda]^{-1}$ dans $\pi_{1}\left(X, w_{1}\right)$.

Soit $\mathbb{T}_{1}$ le tore bordé par $\xi$ contenant $w_{1}$. La géodésique dans la classe d'homotopie libre de $\lambda$ est contenue dans $\mathbb{T}_{1}$. En la découpant on visualise facilement les lacets $\lambda$ et $\iota_{X}(\lambda)$ : ils correspondent aux segments issus de $w_{1}$ orthogonaux aux perpendiculaires communes du pantalon (Figs. 10 et 11). Notons que le produit $\lambda \cdot \iota_{X}(\lambda)^{-1}$ forme une géodésique non simple s'auto-intersectant en $w_{1}$.

Lemme 3.4 La géodésique dans la classe d'homotopie libre de $\lambda$ réalise la systole du tore $\mathbb{T}_{1}$, c'est donc $\alpha_{1}$. 


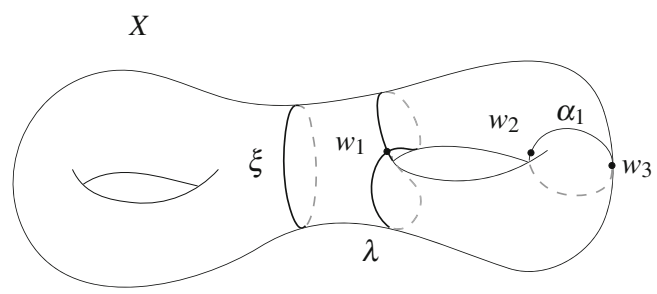

Fig. 10 Lacet réalisant le rayon d'injectivité

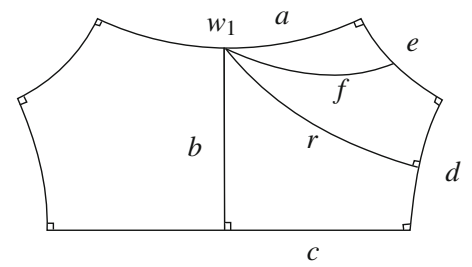

Fig. 11 Notations dans le pentagone droit

Preuve La géodésique dans la classe d'homotopie libre de $\lambda$ est plus petite que n'importe quelle géodésique passant par $w_{1}$, car $\lambda$ réalise le rayon d'injectivité $R_{w_{1}}$. En particulier le tore $\mathbb{T}_{1}$ a exactement une systole, et celle-ci ne passe pas par $w_{1}$. La plus petite géodésique fermée simple après la systole passe par $w_{1}$ (lemme 3.1), ainsi il vient que la géodésique dans la classe d'homotopie libre de $\lambda$ est la systole de $\mathbb{T}_{1}$.

\subsubsection{Principe de la preuve}

Par découpe de $\alpha_{1}$ et de ses perpendiculaires communes avec $\xi$, le tore $\mathbb{T}_{1}$ se divise en deux hexagones droits isométriques. Ces hexagones sont à leur tour formés de deux pentagones droits isométriques. Un de ces pentagones est représenté en Fig. 11 avec la convention : $c=\xi / 4, e=\alpha_{1} / 2, r=R_{w_{1}}$, et $\cosh (f)=\cosh (e / 2) \cosh (a)$. Le segment $f$ relie $w_{1}$ au milieu de $e$, sa longueur majore la demi-longueur de la plus petite géodésique de $\mathbb{T}_{1}$ passant par $w_{1}$. Pour démontrer la proposition 3.1 il suffit de prouver que $\cosh (f)<2,88$.

Nous allons prouver cette inégalité par l'absurde : sous l'hypothèse

$$
\cosh (f) \geq 2,88
$$

nous allons élaborer une procédure prenant en entrée un encadrement de $r$ et sortant une majoration de $\beta_{2}$ et de la plus petite géodésique $\gamma \in \mathcal{G}_{\xi}$ telle que $\gamma_{1}=\alpha_{1}$ et $\gamma_{2}=\beta_{2}$ avec $\left(\gamma_{1}, \gamma_{2}\right)=\Xi(\gamma)$. En implémentant cette procédure sur ordinateur nous verrons que les longueurs de ces géodésiques sont inférieures à $2 \operatorname{arccosh}(4,67)$ pour toutes les valeurs possibles de $r$, ce qui contredira $\operatorname{sys}_{3}>2 \operatorname{arccosh}(4,67)$. 


\subsubsection{Trigonométrie et premières estimations}

Voici trois relations trigonométriques dans (abcde) (voir [8]) :

$$
\begin{aligned}
\cosh (a) & =\frac{\sinh (r)}{\sinh (e)} \\
\cosh (c) & =\tanh (a) \sinh (r) \\
\cosh (f) & =\frac{\sinh (r)}{\sqrt{2(\cosh (e)-1)}} .
\end{aligned}
$$

De ces relations nous pouvons immédiatement tirer quelques estimations :

$$
\begin{aligned}
& \cosh (e)<1,44 \\
& \cosh (a)>2,6 \\
& \cosh (c)>0,923 \sinh (r)
\end{aligned}
$$

La première estimation se déduit de (3.8) et des inégalités (3.5) et (3.1). La deuxième se déduit de la première via $\cosh (a) \cosh (e / 2)=\cosh (f) \geq 2,88$. Enfin la troisième résulte (3.7) et (3.10).

\subsubsection{Procédure de majoration}

Dans ce paragraphe nous présentons une procédure prenant en entrée un encadrement de $r$, et sortant une majoration à la fois de $\beta_{2}$ et de la plus petite géodésique $\gamma \in$ $\mathcal{G}_{\xi}$ vérifiant $\Xi(\gamma)=\left(\alpha_{1}, \beta_{2}\right)$. Cette procédure consiste en un enchaînement d'une dizaine d'inégalités dont le cheminement global se résume ainsi : par des formules de trigonométrie nous encadrons facilement les longueurs de $\xi$ et $\alpha_{1}^{\prime}$, en revanche l'obtention d'inégalités sur $\alpha_{2}, \alpha_{2}^{\prime}, \beta_{2}$ et $\beta_{2}^{\prime}$ s'avère plus délicate, il s'agit d'exprimer la longueur de la plus petite géodésique de $\mathcal{G}_{\xi}$ vérifiant $\Xi(\gamma)=\left(\alpha_{1}, \alpha_{2}\right)$ et d'écrire qu'elle dépasse $2 \operatorname{arccosh}(4,67)$ (on majore facilement $\alpha_{1}$ et $\alpha_{2} \operatorname{par} 2 \operatorname{arccosh}(4,67)$ ).

Partons d'un encadrement

$$
r^{(1)} \leq r \leq r^{(2)}
$$

1. De ce dernier et de (3.7) nous tirons un encadrement $c^{(1)} \leq c \leq c^{(2)}$. Par (3.4) nous supposerons $1,111<c^{(1)}$.

2. De $\sinh b=\cosh e / \sinh c$ et $\cosh b=1 /(\tanh a \tanh c)$ vient $b \leq b^{(2)}$ avec

$$
b^{(2)}=\min \left(\operatorname{arcsinh}\left(\frac{1,44}{\sinh c^{(1)}}\right), \operatorname{arccosh}\left(\frac{1}{\tanh (1,609) \tanh c^{(1)}}\right)\right) .
$$

3. Les systoles $\alpha_{1}$ et $\alpha_{2}$ des tores $\mathbb{T}_{1}$ et $\mathbb{T}_{2}$ sont inférieures à $2 \operatorname{arccosh}(4,67)$; c'est clair pour $\alpha_{1}$ car $\alpha_{1} / 2=e$ et $\cosh (e)<1.44$ (3.9), pour $\alpha_{2}$ il convient d'appliquer (3.3) avec la majoration de $\xi$ donnée par $\cosh (\xi / 4)<\sinh r$ (3.7). 
Nous en déduisons que la plus petite géodésique $\gamma \in \mathcal{G} \xi$ vérifiant $\Xi(\gamma)=\left(\alpha_{1}, \alpha_{2}\right)$ est nécessairement de longueur supérieure à $2 \operatorname{arccosh}(4,67)$. De ce fait $\alpha_{2}^{\prime}$ est minorée $\operatorname{par} \alpha_{2}^{\prime(1)}$, le double de la solution positive de l'équation (voir $§ 2.4$ )

$$
\cosh b^{(2)} \cosh x \cosh c^{(2)}+\sinh b^{(2)} \sinh x=4,67 .
$$

La majoration (3.2) s'appliquant directement à $\alpha_{2}^{\prime}$, nous obtenons finalement un encadrement $\alpha_{2}^{\prime(1)} \leq \alpha_{2}^{\prime} \leq \alpha_{2}^{\prime(2)}$.

4. Soit $\mu$ la distance sur $\xi$ entre $\alpha_{1}^{\prime}$ et $\alpha_{2}^{\prime}$. En suivant le raisonnement précédent, et en usant de (3.4) et (3.2), nous avons $\mu^{(1)} \leq \mu$ où $\mu^{(1)}$ est la solution positive de l'équation

$$
\cosh b^{(2)} \sqrt{1+\frac{1}{4 \sinh ^{2}\left(c^{(1)} / 3\right)}} \cosh x+\sinh b^{(2)} \frac{1}{2 \sinh \left(c^{(1)} / 3\right)}=4,67 .
$$

5. La relation $\cosh \left(\alpha_{2} / 2\right)=\sinh \left(\alpha_{2}^{\prime} / 2\right) \sinh c$ (voir $\left.\S 2.2\right)$ donne un encadrement $\alpha_{2}^{(1)} \leq \alpha_{2} \leq \alpha_{2}^{(2)}$, dont nous améliorons éventuellement le majorant par (3.3).

6. Soit $a_{2}$ la perpendiculaire commune à $\alpha_{2}$ et $\alpha_{2}^{\prime}$, nous avons $a_{2} \leq a_{2}^{(2)}$ avec

$$
a_{2}^{(2)}=\min \left(\operatorname{arctanh}\left(\frac{1}{\cosh \left(\alpha_{2}^{\prime(1)} / 2\right) \tanh c^{(1)}}\right), \operatorname{arccosh}\left(\frac{1}{\tanh \left(\alpha_{2}(1) / 2\right) \tanh \left(\alpha_{2}^{\prime(1)} / 2\right)}\right)\right) .
$$

7. De $\cosh \left(\beta_{2} / 2\right) \leq \cosh \left(\alpha_{2} / 4\right) \cosh a_{2}$ nous tirons une majoration $\beta_{2} \leq \beta_{2}^{(2)}$.

8. De la relation $\cosh \left(\beta_{2} / 2\right)=\sinh \left(\beta_{2}^{\prime} / 2\right) \sinh c$ nous déduisons une majoration $\beta_{2}^{\prime} \leq \beta_{2}^{\prime(2)}$.

9. Nous majorons par $\gamma^{(2)}$ la longueur de la plus petite géodésique $\gamma \in \mathcal{G}_{\xi}$ telle que $\Xi(\gamma)=\left(\alpha_{1}, \beta_{2}\right)$, avec $\gamma^{(2)}$ définie par

$$
\begin{aligned}
\cosh \left(\frac{\gamma^{(2)}}{2}\right)= & \cosh b^{(2)} \cosh \left(\frac{\beta_{2}^{\prime(2)}}{2}\right) \cosh \left(\frac{4 c^{(2)}}{3}-\mu^{(1)}\right) \\
& +\sinh b^{(2)} \sinh \left(\frac{\beta_{2}^{\prime(2)}}{2}\right) .
\end{aligned}
$$

Le paramètre $4 c^{(2)} / 3-\mu^{(1)}$ correspond au twist, c'est-à-dire à la distance entre $\alpha_{1}^{\prime}$ et $\beta_{2}^{\prime}$ sur $\xi$. Comme la distance sur $\xi$ entre $\alpha_{2}^{\prime}$ et $\beta_{2}^{\prime}$ est supérieure ou égale à $\xi / 6$ (remarque 3.2), et celle entre $\alpha_{1}^{\prime}$ et $\alpha_{2}^{\prime}$ est minoré par $\mu^{(1)}$, nous trouvons que le twist est inférieure à $\xi / 2-\xi / 6-\mu^{(1)}$. 
Voici le code de la procédure en Maple :

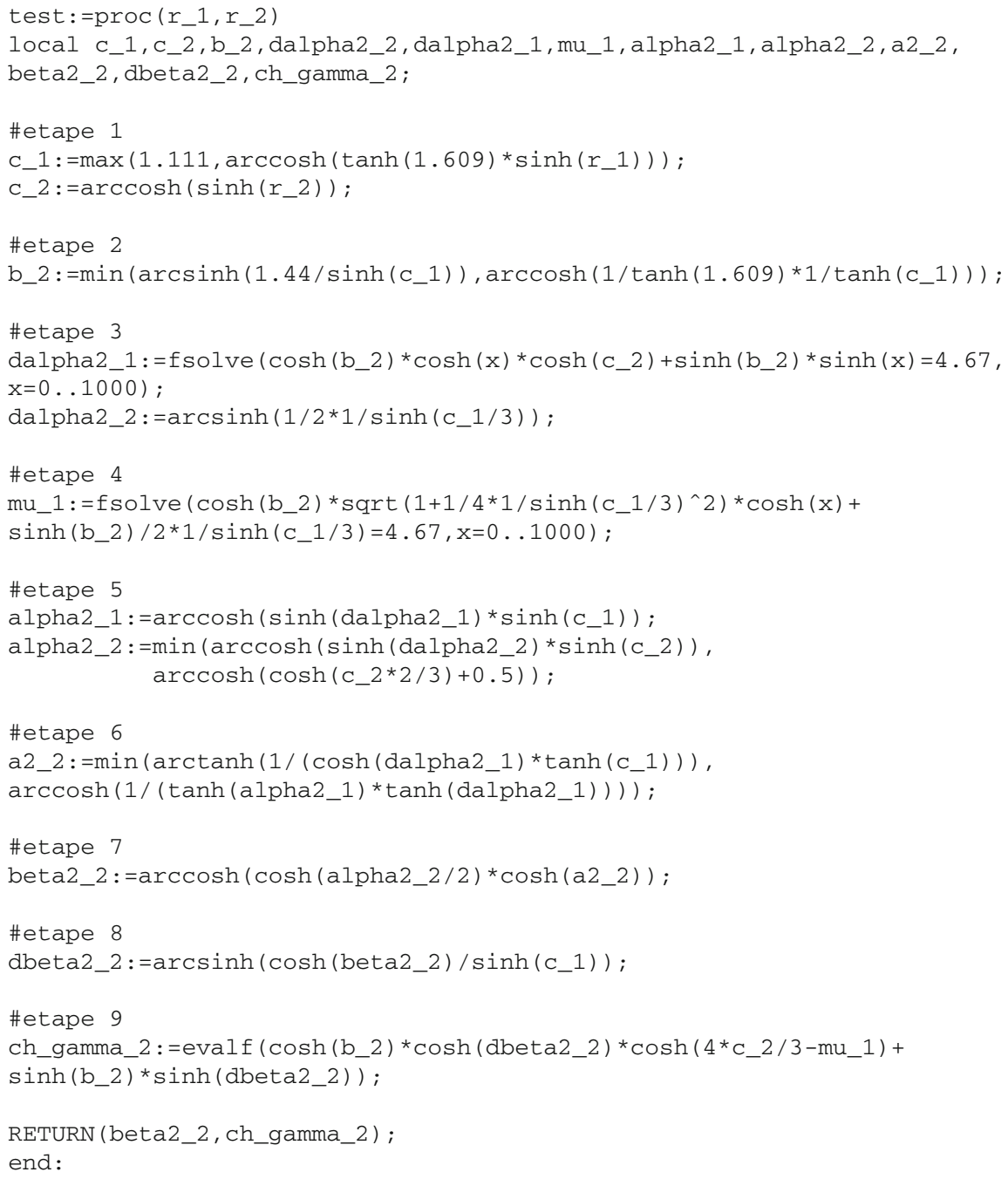

\subsubsection{Conclusion}

Nous pouvons supposer $r \in[1,292 ; 1,72]$, la minoration se déduit de (3.7) et (3.4), et la majoration vient directement de (3.1). En appliquant la procédure à une subdivision suffisamment fine de cet intervalle, il appparaît que la plus courte géodésique $\gamma \in \mathcal{G}_{\xi}$ telle que $\Xi(\gamma)=\left(\alpha_{1}, \beta_{2}\right)$ est de longueur inférieure à $2 \operatorname{arccosh}(4,67)$. Les géodésiques $\alpha_{1}$ et $\beta_{2}$ étant elles aussi de longueurs inférieures à $2 \operatorname{arccosh}(4,67)$ (la majoration de $\beta_{2}$ est donnée par la procédure), l'hypothèse $\operatorname{sys}_{3} \geq 2 \operatorname{arccosh}(4,67)$ est contredite. Ceci termine la preuve de la proposition 3.1. 


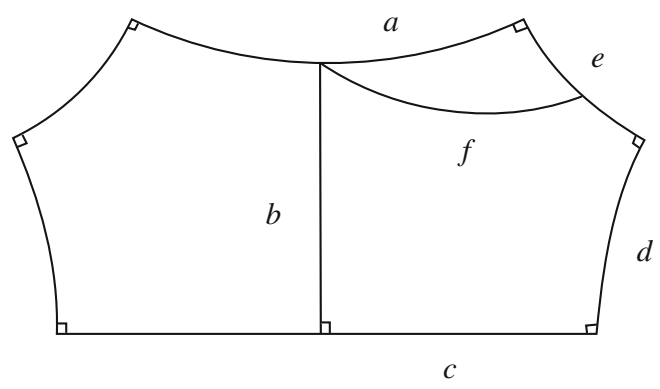

Fig. 12 Notations dans le pentagone droit

\section{La 3-systole est la systole de la famille $\mathcal{G}_{\xi} \cup\{\xi\}$}

Soit $X$ une surface de Riemann de genre 2 vérifiant $\operatorname{sys}_{3}(X) \geq 2 \operatorname{arccosh}(4,67)$. D'après le corollaire 1.1 , tous les sous-graphes minimaux de $G_{c o n t}(X)$ sont isomorphes au graphe $G_{9}$, en particulier $G_{c o n t}(X)$ a exactement deux composantes connexes, et il existe une unique géodésique séparante $\xi$ disjointe de $G_{\text {cont }}(X)$.

Dans cette partie nous allons établir une égalité entre la 3-systole de $X$ et la systole de la famille $\mathcal{G}_{\xi} \cup\{\xi\}$. Cela se fera en deux étapes, dans un premier temps nous regarderons quelles sont les plus petites géodésiques de $\mathcal{G}_{\xi} \cup\{\xi\}$, nous montrerons ensuite que si $\gamma$ est l'une d'elles alors la longueur de la partition $\left(\gamma, \gamma_{1}, \gamma_{2}\right)$ est donnée par la longueur de $\gamma$.

Notations Nous désignerons par $\mathcal{F}$ la famille de géodésiques

$$
\mathcal{F}=\left\{\gamma \in \mathcal{G}_{\xi} ; \Xi(\gamma) \in\left\{\alpha_{1}, \beta_{1}, \delta_{1}\right\} \times\left\{\alpha_{2}, \beta_{2}, \delta_{2}\right\}\right\} \cup\{\xi\}
$$

Nous associerons à tout élément $\gamma$ de $\mathcal{G}_{\xi}$ la partition $\left(\gamma, \gamma_{1}, \gamma_{2}\right)$, et à $\xi$ la partition $\left(\xi, \alpha_{1}, \alpha_{2}\right)$. Nous avons vu au $\$ 2.4$ que la longueur d'une géodésique $\gamma \in \mathcal{G}_{\xi}$ est donnée par $\cosh (\gamma / 2)=\cosh \left(\gamma_{1}^{\prime} / 2\right) \cosh \left(\gamma_{2}^{\prime} / 2\right) \cosh (\mu)+\sinh \left(\gamma_{1}^{\prime} / 2\right) \sinh \left(\gamma_{2}^{\prime} / 2\right)$, où $\mu$ est, modulo $\xi$, la longueur d'un des quatres segments de $\xi$ délimités par les points d'intersection de $\gamma_{1}^{\prime}$ et $\gamma_{2}^{\prime}$ avec $\xi$.

Nous travaillerons fréquemment dans le pentagone (abcde), moitié de l'hexagone droit issu de la découpe de $\beta_{1}$ dans le tore $\mathbb{T}_{1}$. Ce pentagone est représenté en Fig. 12 avec les conventions $b=\beta_{1}^{\prime} / 2, c=\xi / 4, e=\beta_{1} / 2$ et $f=\delta_{1} / 2$.

4.1 Deux lemmes techniques

Lemme 4.1 Dans le pentagone (abcde) on a $\cosh (e)<2,16$ ou $\cosh (a)<1,62$.

Preuve Supposons par l'absurde $2,16 \leq \cosh (e)$ et $1,62 \leq \cosh (a)$. Alors, dans (abcde) nous avons les cinq inégalités suivantes : 


$$
\begin{aligned}
2,16 \leq \cosh (e) & \leq 2,88, \\
1,62 \leq \cosh (a) & \leq 2,88, \\
2,44 \leq \cosh (c) & \leq 8, \\
\cosh (b) & \leq 1,4, \\
\sinh (b) & \leq \frac{2,88}{\sinh (c)} .
\end{aligned}
$$

Les deux majorations par 2, 88 proviennent de la borne sur la longueur des arêtes de $G_{\text {cont }}(X)$. En utilisant l'identité $\cosh (c)=\sinh (a) \sinh (e)$ avec les deux premiers encadrements nous obtenons le troisième encadrement. La majoration de $\cosh (b)$ se déduit des deux premières minorations et de l'égalité $\tanh (e) \cosh (a) \tanh (b)=1$. Enfin, la dernière inégalité est triviale.

Nous allons exhiber une géodésique $\eta \in \mathcal{F} \backslash\{\xi\}$ telle que la longueur de la partition $\left(\eta, \eta_{1}, \eta_{2}\right)$ est donnée par $\eta$ et inférieure à $2 \operatorname{arccosh}(4,67)$. Partons de $\eta \in \mathcal{F} \backslash\{\xi\}$ vérifiant $\Xi(\eta) \in\left\{\alpha_{1}, \beta_{1}\right\} \times\left\{\alpha_{2}\right\}$ et $\mu \leq \xi / 6$, un tel $\eta$ existe par le lemme 3.2. En majorant le rayon $\alpha_{2}^{\prime}$ par (3.2) nous avons

$$
\begin{aligned}
& \cosh (\eta / 2)=\cosh \left(\beta_{1}^{\prime} / 2\right) \cosh \left(\alpha_{2}^{\prime} / 2\right) \cosh (\mu)+\sinh \left(\beta_{1}^{\prime} / 2\right) \sinh \left(\alpha_{2}^{\prime} / 2\right) \\
& \cosh (\eta / 2) \leq \cosh \left(\beta_{1}^{\prime} / 2\right) \sqrt{1+\frac{1}{4 \sinh (\xi / 12)^{2}}} \cosh (\xi / 6)+\sinh \left(\beta_{1}^{\prime} / 2\right) \frac{1}{2 \sinh (\xi / 12)}
\end{aligned}
$$

Nous allons distinguer quatre cas correspondant à des intervalles de valeurs de $\xi$. Ces intervalles sont déterminés par $\cosh (\xi / 4) \in[2,44 ; 3]$, [3 ; 4], [4; 6], [6 ; 8]. Pour chaque encadrement de $\xi$ nous fournissons une majoration de $\beta_{1}^{\prime}$, nous contrôlons ainsi la longueur de $\eta$. Dans les deux premiers cas, nous utilisons l'inégalité $\cosh (b) \leq 1,4$ pour majorer $\beta_{1}^{\prime}$, nous trouvons $\cosh (\eta) \leq 4,31$ dans le premier cas et $\cosh (\eta) \leq$ 4, 57 dans le deuxième. Dans les deux derniers cas, nous nous servons de l'inégalité $\sinh (b) \leq \frac{2,88}{\sinh (c)}$ pour majorer $\beta_{1}^{\prime}$, nous trouvons $\cosh (\eta) \leq 4,57$ dans le troisième et $\cosh (\eta) \leq 4,37$ dans le quatrième.

Dans tous les cas, la géodésique $\eta$ vérifie bien $\cosh (\eta / 2)<4$, 67. Les géodésiques $\eta_{1}$ et $\eta_{2}$ sont de longueurs inférieures à $2 \operatorname{arccosh}(2,88)$ car elles se projettent sur des arêtes du graphe de contiguïté. Finalement, on a bien une absurdité car la partition $\left(\eta, \eta_{1}, \eta_{2}\right)$ contredit l'hypothèse $\cosh \left(\operatorname{sys}_{3}(X) / 2\right) \geq 4,67$.

Conséquence 4.2 La systole $\alpha_{1}$ du tore $\mathbb{T}_{1}$ vérifie $\cosh \left(\alpha_{1} / 2\right)<2$, 26. Dans le cas où $\cosh (e)<2,16$ c'est évident puisque $\alpha_{1}$ est plus courte que $\beta_{1}$, dans le cas où $\cosh (a)<1,62$ on utilise les majorations $\cosh \left(\beta_{1} / 2\right) \leq 2,88$ et $\cosh \left(\alpha_{1} / 2\right) \leq$ $\cosh \left(\beta_{1} / 4\right) \cosh (a)$.

Remarque 4.1 Ces inégalités sont aussi valables dans le tore $\mathbb{T}_{2}$.

Lemme 4.2 La longueur d'une partition $\left(\gamma, \gamma_{1}, \gamma_{2}\right)$ associée à un élément $\gamma$ de $\mathcal{F}$ est donnée par la longueur de $\gamma$. 
Preuve Soit $\gamma$ un élément de $\mathcal{F}$. Ou bien la longueur de la partition $\left(\gamma, \gamma_{1}, \gamma_{2}\right)$ est égale à la longueur de $\gamma$, c'est par exemple le cas lorsque $\gamma_{1}, \gamma_{2}$ se projettent sur des arêtes du graphe de contiguïté (proposition 3.1). Ou bien la longueur de la partition est donnée par la longueur du couple $\left(\gamma_{1}, \gamma_{2}\right)$. Comme les $\alpha_{i}$ et $\beta_{i}$ se projettent sur des arêtes de $G_{\text {cont }}(X)$, on a $\gamma_{1}=\delta_{1}$ ou $\gamma_{2}=\delta_{2}$. Pour fixer les idées, nous supposerons que la longueur de la partition est donnée par $\gamma_{1}=\delta_{1}$. Dans le pentagone (abcde) nous avons alors :

$$
\left.\begin{array}{rl}
\cosh (a) \cosh (e) & \geq \cosh \left(\delta_{1} / 2\right) \geq 4,67 \\
\cosh (e) & \geq \cosh \left(\alpha_{1} / 2\right) \geq \cosh (a)
\end{array}\right\} \Longrightarrow \cosh (e) \geq \sqrt{4,67}>2,16
$$

et de même,

$$
\left.\begin{array}{rl}
\cosh (a) \cosh (e) & \geq \cosh \left(\delta_{1} / 2\right) \geq 4,67 \\
2,88 & \geq \cosh (e)
\end{array}\right\} \Longrightarrow \cosh (a) \geq \frac{4.67}{2.88}>1,62 .
$$

Ces minorations contredisent le lemme précédent.

\subsection{Egalité entre la 3-systole et la systole de la famille $\mathcal{G}_{\xi} \cup\{\xi\}$}

C'est le dénouement, nous allons abandonner la 3-systole au profit de la systole de la famille $\mathcal{G}_{\xi} \cup\{\xi\}$.

Lemme 4.3 Soit $\gamma$ une géodésique réalisant la systole de la famille $\mathcal{G}_{\xi}$, alors

$$
\Xi(\gamma) \in\left\{\alpha_{1}, \beta_{1}, \delta_{1}, \varepsilon_{1}\right\} \times\left\{\alpha_{2}, \beta_{2}, \delta_{2}, \varepsilon_{2}\right\}
$$

Preuve Soit $\gamma$ une géodésique de longueur minimale dans la famille $\mathcal{G}_{\xi}$. En raison de leurs propriétés de minimisation les géodésiques $\gamma, \alpha_{i}, \beta_{i}$ ne peuvent s'intersecter en dehors des points de Weierstrass. Comme $\alpha_{i}, \beta_{i}, \delta_{i}$ forment un triangle, nous avons l'alternative suivante : ou bien $\gamma$ ne rencontre pas le triangle $\left(\alpha_{i} \beta_{i} \delta_{i}\right)$ en dehors de ses sommets, alors $\gamma$ est disjointe d'une des trois géodésiques $\alpha_{i}, \beta_{i}, \delta_{i}$; ou bien $\gamma$ rencontre $\delta_{i}$ en dehors de ses points de Weierstrass, alors $\gamma$ coupe $\delta_{i}$ en exactement deux points (échangés par l'involution hyperelliptique) et passe par le point de Weierstrass opposé, dans ce cas $\gamma$ est disjointe de $\varepsilon_{i}$, autrement dit $\varepsilon_{i} \in \Xi(\gamma)$.

Rappelons nos hypothèses : $X$ est une surface de Riemann de genre 2 dont la 3 -systole vérifie sys $_{3} \geq 2 \operatorname{arccosh}(4,67)$.

Proposition 4.1 Soit $\gamma$ une géodésique réalisant la systole de la famille $\mathcal{G}_{\xi} \cup\{\xi\}$, alors $\Xi(\gamma) \in\left\{\alpha_{1}, \beta_{1}, \delta_{1}\right\} \times\left\{\alpha_{2}, \beta_{2}, \delta_{2}\right\}$.

Preuve Il s'agit de montrer que le deuxième cas de l'alternative de la démonstration précédente ne peut se produire. Supposons par l'absurde que $\gamma$ coupe $\delta_{1}$ en dehors des points de Weierstrass. En découpant la géodésique $\alpha_{1}$ dans le tore $\mathbb{T}_{1}$ nous obtenons un pantalon hyperbolique (Fig. 13). Ce pantalon se scinde en deux hexagones droits isométriques, se divisant à leurs tours en pentagones droits isométriques. Nous allons 


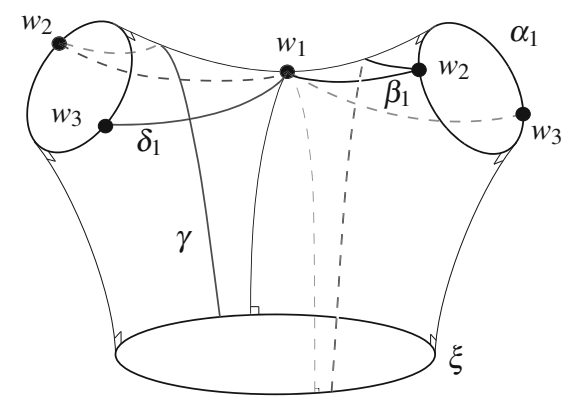

Fig. 13 Configuration dans le pantalon

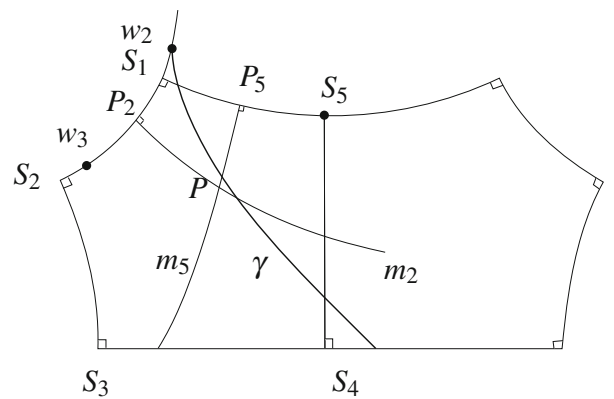

Fig. 14 Configuration dans l'hexagone

travailler dans un de ces pentagones, dont nous noterons $S_{i}(i=1, \ldots, 5)$ les sommets (Fig. 14). Soient $m_{5}$ et $m_{2}$ les médiatrices respectives des segments $\left[S_{1}, S_{5}\right]$ et $\left[w_{2}, w_{3}\right]$, la longueur de chacun de ces segments est inférieure à $\operatorname{arccosh}(2,88)$, ainsi $\sinh \left(P_{2} S_{1}\right) \sinh \left(S_{1} P_{5}\right)<1$ et nécessairement $m_{2}$ et $m_{5}$ s'intersectent en un point $P$ de $X$.

Plaçons nous dans le revêtement universel $\tilde{X}$ de $X$, et considérons $\tilde{M}$ un point de $\tilde{\gamma}$ se situant au-delà du trirectangle $\left(\tilde{P}_{2} \tilde{P} \tilde{P}_{5} \tilde{S}_{1}\right)$. Par construction ce point est soit plus proche de $\tilde{S}_{5}=\tilde{w}_{1}$ que de $\tilde{S}_{1}$, soit plus proche de $\tilde{w}_{3}$ que de $\tilde{w}_{2}$, dans les deux cas $\tilde{M}$ est plus proche d'un des deux relevés des points de Weierstrass $w_{1}, w_{3}$ que du relevé de $w_{2}$. Ceci contredit presque la minimalité de $\gamma$ dans la famille $\mathcal{G}_{\xi}$, le seul problème étant qu'on peut a priori parcourir plusieurs fois $\gamma$ dans le revêtement universel avant de sortir du trirectangle.

Montrons que la distance de $\tilde{P}$ à $\tilde{w}_{2}$ est inférieure en cosinus hyperbolique à 2,88 . Nous avons $\cosh \left(\tilde{P} \tilde{w}_{2}\right)=\cosh \left(\tilde{P}_{2} \tilde{w}_{2}\right) \cosh \left(\tilde{P}_{2} \tilde{P}\right), \operatorname{mais} \cosh \left(\tilde{P}_{2} \tilde{w}_{2}\right)=\cosh \left(\alpha_{1} / 4\right)<$ 1, 28 par la conséquence 4.2 . Il suffit donc de majorer $\cosh \left(\tilde{P}_{2} \tilde{P}\right)$. Soit $\phi$ l'angle du trirectangle en $\tilde{P}$, par les formules usuelles il vient :

$$
\left\{\begin{array}{l}
\cos \phi=\sinh \left(\tilde{P}_{2} \tilde{S}_{1}\right) \sinh \left(\tilde{S}_{1} \tilde{P}_{5}\right) \leq \sinh \left(\alpha_{1} / 4\right) \sinh \left(\beta_{1} / 4\right) \\
\cosh \left(\tilde{P}_{2} \tilde{P}\right)=\frac{\cosh \left(\tilde{S}_{1} \tilde{P}_{5}\right)}{\sin \phi} \leq \frac{\cosh \left(\beta_{1} / 4\right)}{\sin \phi}
\end{array}\right.
$$


Par le lemme 4.1 on a $\cosh \left(\alpha_{1} / 2\right) \leq 2,26$ et $\cosh \left(\beta_{1} / 2\right) \leq 2,88$, ou $\cosh \left(\alpha_{1} / 2\right) \leq$ 2,16 et $\cosh \left(\beta_{1} / 2\right) \leq 2,16$. Dans le premier cas nous trouvons $\cosh \left(\tilde{P}_{2} \tilde{P}\right) \leq 2$, 2 et dans le deuxième $\cosh \left(\tilde{P}_{2} \tilde{P}\right) \leq 1,55$. Au final nous avons bien $\cosh \left(\tilde{P} \tilde{w}_{2}\right)<2,88$.

La longueur de $\gamma / 2$ est supérieure à 2,88 en cosinus hyperbolique, sinon nous pourrions contredire la proposition 1.1. La géodésique $\gamma$ part de $w_{2}$ pour pénétrer dans le pentagone $\left(S_{1} S_{2} S_{3} S_{4} S_{5}\right)$, et traverse alors le trirectangle $\left(P_{2} P P_{5} S_{1}\right)$. Vue notre majoration de la distance $d\left(P, w_{2}\right), \gamma$ sort du tirectangle avant d'atteindre son deuxième point de Weierstrass ; ainsi, les points de $\gamma$ à la sortie du trirectangle sont plus proches de $w_{1}$ ou de $w_{3}$ que de $w_{2}$. C'est absurde puisque $\gamma$ est supposée de longueur minimale dans $\mathcal{G}_{\xi}$.

Théorème 4.1 Lorsque la 3-systole vérifie $\operatorname{sys}_{3} \geq 2 \operatorname{arccosh}(4,67)$, la systole de la famille $\mathcal{G}_{\xi} \cup\{\xi\}$ est égale à la 3 -systole.

Preuve Nous venons de voir que lorsque $\cosh \left(\operatorname{sys}_{3}(X) / 2\right) \geq 4,67$ la systole de $\mathcal{G}_{\xi} \cup\{\xi\}$ est égale à la systole de $\mathcal{F}$. Mais la longueur d'une partition associée à une géodésique de $\mathcal{F}$ est égale à la longueur de la géodésique, ainsi la systole de $\mathcal{F}$ coïncide avec la longueur minimale des partitions associées aux éléments de $\mathcal{F}$. Donc

$$
\operatorname{sys}_{\mathcal{G}_{\xi} \cup\{\xi\}}=\operatorname{sys}_{\mathcal{F}}=\inf _{\gamma \in \mathcal{F}} l(\gamma)=\inf _{\gamma \in \mathcal{F}} l\left(\gamma, \gamma_{1}, \gamma_{2}\right) \geq \operatorname{sys}_{3},
$$

la 3-systole est majorée par la systole de la famille $\mathcal{G}_{\xi} \cup\{\xi\}$.

Supposons par l'absurde que la 3-systole n'est pas réalisée par la systole de $\mathcal{G}_{\xi} \cup\{\xi\}$. Considérons une partition de longueur minimale $\left(\gamma_{1}, \gamma_{2}, \gamma_{3}\right)$, une des trois géodésiques de cette partition (disons $\gamma_{1}$ ) intersecte nécessairement $\xi$ en au moins quatre points, car le nombre de points d'intersection est pair et seules les géodésiques de $\mathcal{G}_{\xi}$ intersectent $\xi$ en exactement deux points. À partir de cette géodésique nous allons construire une partition de longueur inférieure à $\gamma_{1}$, ce qui est absurde. Nous distinguons deux cas :

Cas où $\gamma_{1}$ est non séparante. Comme $\gamma_{1}$ coupe au moins deux fois chaque composante du graphe de contiguïté, elle supporte au moins quatre segments intersectant l'une des composantes du graphe en un point de Weierstrass et l'autre à l'intérieur d'une des arêtes. Prenons le plus petit de ces segments, nous le complétons par un bout d'arête en un chemin géodésique par morceaux passant par un point de Weierstrass de chacune des composantes. La longueur de ce chemin est inférieure à $\gamma_{1} / 4+\operatorname{arccosh}(2,88) / 2$ et donc inférieure à $\gamma_{1} / 2$. En prenant le double de ce chemin par l'involution hyperelliptique nous obtenons un lacet simple, la géodésique dans sa classe d'homotopie libre appartient à $\mathcal{G}_{\xi}$ et est en longueur strictement inférieure à $\gamma_{1}$, absurde car sys $\mathcal{G}_{\xi} \cup \xi>l\left(\gamma_{1}\right)$.

Cas où $\gamma_{1}$ est séparante. La géodésique $\gamma_{1}$ intersecte chaque composante du graphe de contiguïté, pour fixer les idées nous supposerons qu'elle intersecte $\alpha_{1}$, ce choix n'ayant aucune incidence sur la suite. La géodésique $\gamma_{1}$ est séparante et divise la surface $X$ en deux tores à un bord. Si $\alpha_{1}$ intersecte $\gamma_{1}$ en au moins quatre points, on considère une systole de chaque tore bordé par $\gamma_{1}$. Les rayons duaux de ces systoles sont plus courts que les segments de $\alpha_{1}$ reliant deux points de $\gamma_{1}$, en particulier la somme de ces deux rayons est inférieure à $\alpha_{1} / 2$. À partir de ces rayons duaux et de $\gamma_{1}$, on construit une géodésique disjointe des systoles et de longueur inférieure à 
$\alpha_{1} / 2+\gamma_{1} / 2$, donc inférieure à $\gamma_{1}$. Cette géodésique forme avec les deux systoles une partition de longueur inférieure à $\gamma_{1}$, absurde. Si $\alpha_{1}$ intersecte $\gamma_{1}$ en exactement deux points, alors les deux géodésiques disjointes de $\gamma_{1}$ et $\alpha_{1}$ sont de longueurs inférieures à $\gamma_{1}$. En effet, les rayons duaux de ces géodésiques sont de longueurs inférieures à $\alpha_{1}$, or, en se plaçant dans des pentagones, on exprime les longueurs de ces géodésiques en fonction des longueurs des rayons duaux et de $\gamma_{1}$. Donc ces deux géodésiques forment avec $\alpha_{1}$ une partition de longueur inférieure à $\gamma_{1}$, absurde.

\section{Existence et détermination du maximum global de sys $_{3}$}

\subsection{Existence}

Proposition 5.1 Les surfaces de Riemann de genre 2 dont la 3-systole est supérieure ou égale à 2 arccosh $(4,67)$ forment un compact de l'espace des modules.

Preuve Cet ensemble est un fermé de l'espace des modules, il s'agit de montrer qu'il n'atteint pas l'infini. Rappelons que nous allons à l'infini dans l'espace des modules lorsque la systole tend vers 0 [12]. Supposons l'existence d'une surface $X$ avec $\cosh \left(\operatorname{sys}_{3}(X) / 2\right) \geq 4,67$ et sys $(X)$ aussi proche de 0 que voulu. Vu (3.4) les systoles de $X$ ne sont pas séparantes, elles sont donc associées à des arêtes du graphe de contiguïté. Disons par exemple que $\alpha_{1}$ est une systole, alors les inégalités $1 \leq$ $\cosh (\xi / 4) \leq \sinh \left(\alpha_{1} / 2\right) \sinh \left(\beta_{1} / 2\right) \leq 2.8 \sinh \left(\alpha_{1} / 2\right)$ entraînent une contradiction lorsque $\alpha_{1}$ tend vers 0 .

Corollaire 5.1 La 3-systole admet un maximum global sur $\mathcal{T}_{2}$.

\subsection{Détermination}

Notations Nous désignerons par $M$ un point de $\mathcal{T}_{2}$ et par X la surface sous-jacente. Nous travaillerons dans l'ouvert $D$ de $\mathcal{T}_{2}$ défini par $D=\left\{\operatorname{sys}_{3}>2 \operatorname{arccosh}(4,67)\right\}$.

Nous avons :

$$
B(2)=\max _{\bar{D}} \operatorname{sys}_{3}=\max _{\bar{D}} \operatorname{sys}_{\mathcal{G}_{\xi} \cup\{\xi\}} \cdot
$$

Les points réalisant le maximum global sur $\bar{D}$ de la fonction $\operatorname{sys}_{\mathcal{G}_{\xi} \cup\{\xi\}}$ appartiennent à $D$. Nous allons rechercher ces points.

\subsubsection{Théorie de Voronoï géométrique}

La systole des surfaces de Riemann est un analogue de l'invariant d'Hermite des réseaux euclidiens. Un cadre théorique général englobant ces deux invariants a été élaboré par C. Bavard [3,5], les notions de perfection et d'eutaxie y sont définies en termes de gradient des fonctions longueur, et l'on retrouve un analogue du théorème de Voronoï (d'abord obtenu par P. Scmutz Schaller dans [15]) dont voici un énoncé dans le cadre qui nous convient : 
Théorème Une surface de Riemann compacte est extrême si et seulement si elle est parfaite et eutactique.

En particulier, les maxima locaux des fonctions de type systole admettent au moins la dimension du Teichmüller plus une géodésiques réalisant la systole (voir aussi [15]). Ici on considère la systole de la famille $\mathcal{G}_{\xi} \cup \xi$, les fonctions longueur de géodésique étant strictement convexes le long des géodésiques de la métrique de Weil-Petersson, il vient que cette fonction rentre bien dans le cadre défini par Bavard dans les $\S \S 1.1$ et 1.2 de [5]. Ainsi, dans une surface réalisant un maximum local de la 3-systole sur $D$, ou de manière équivalente de $\operatorname{sys}_{\mathcal{G}_{\xi} \cup \xi}$ sur $D$, il y a au moins 7 géodésiques de $\mathcal{G}_{\xi} \cup \xi$ de longueur minimale. La géodésique $\xi$ étant potentiellement l'une d'elles, il reste au moins 6 géodésiques de $\mathcal{G}_{\xi}$ de longueur minimale dans $\mathcal{G}_{\xi} \cup \xi$. Rappelons que ces géodésiques appartiennent à $\mathcal{F}$ par le lemme 4.3. Nous allons déterminer les configurations possibles pour ces géodésiques.

Notations Nous noterons $\left(\gamma_{j}\right)_{j \in J}$ la famille des géodésiques distinctes de $\xi$ de longueur minimale dans $\mathcal{F}$.

\subsubsection{Deux lemmes d'intersection}

Lemme 5.1 Les éléments de $\mathcal{F}$ n'intersectent les $\alpha_{i}, \beta_{i}$ et $\delta_{i} q u$ 'en des points de Weierstrass.

Preuve Les éléments de $\mathcal{F} \backslash\{\xi\}$ s'obtiennent essentiellement à partir des rayons duaux $\alpha_{i}^{\prime}, \beta_{i}^{\prime}$ et $\delta_{i}^{\prime}$. Or ces rayons n'intersectent les triangles $\left(\alpha_{i} \beta_{i} \delta_{i}\right)$ qu'en leurs sommets.

Lemme 5.2 Les géodésiques $\left(\gamma_{j}\right)_{j \in J}$ ne s'intersectent pas en dehors des points de Weierstrass.

Preuve Les $\gamma_{j}$ sont toutes de même longueur, la longueur minimale d'un élément de $\mathcal{F}$. Si deux d'entre elles s'intersectaient en dehors des points de Weierstrass, nous pourrions facilement en construire une troisième plus petite.

\subsection{3 Élimination de configurations}

Soit $M_{\max }$ un point de $D$ réalisant un maximum local de la fonction sys $_{\mathcal{F}}$, nous noterons $X_{\max }$ la surface sous-jacente.

Proposition 5.2 Dans $X_{\max }$, deux géodésiques de $\left(\gamma_{j}\right)_{j \in J}$ ne passent jamais par les deux mêmes points de Weierstrass.

Preuve Soient $\gamma_{j_{1}}$ et $\gamma_{j_{2}}$ deux éléments de $\left(\gamma_{j}\right)_{j \in J}$ passant par les mêmes points de Weierstrass. Ces géodésiques diffèrent nécessairement d'un demi-twist selon $\xi$. Ainsi, vue la formule du $\S 2.4$, il est clair que le coefficient de twist selon $\xi$ dans la partition formée de $\xi$ et des géodésiques de $\Xi\left(\gamma_{j_{1}}\right)$ est égal à $1 / 4$.

En découpant $\gamma_{j_{1}}$ et les géodésiques de $\Xi\left(\gamma_{j_{1}}\right)$ dans $X$, nous obtenons deux pantalons hyperboliques, envoyés l'un sur l'autre par l'involution hyperelliptique. Les contraintes données par les lemmes 5.1 et 5.2 permettent de situer toutes les $\gamma_{j}(j \in J)$, elles sont exactement au nombre de 6 , et passent chacune par un des deux points de 


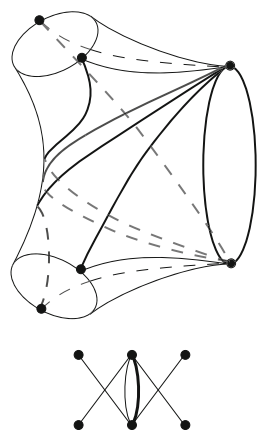

Fig. 15 Configuration topologique de $N(3)$

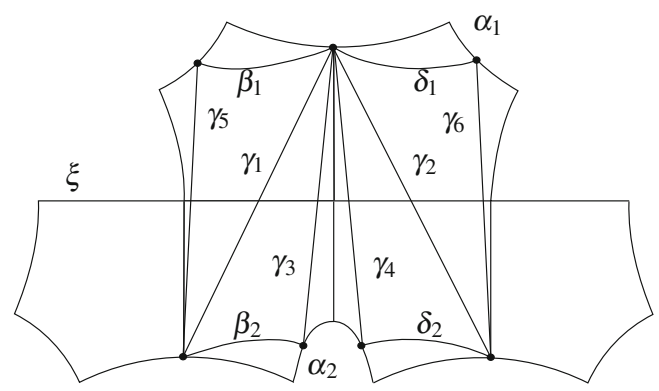

Fig. 16 Patron de $N(3)$

Weierstrass appartenant à $\gamma_{j_{1}}$. Nous avons représenté en Fig. 15 un de ces pantalons, les géodésiques en trait fin correspondent aux $\alpha_{i}, \beta_{i}, \delta_{i}(i=1,2)$, celles en trait gras aux $\gamma_{j}(j \in J)$. Le graphe en-dessous donne un point de vue combinatoire, l'arête en gras s'identifie à $\gamma_{j_{1}}$. Dans la suite de la preuve nous prendrons $J=\{1, \ldots, 6\}$ et $j_{1}=1, j_{2}=2$.

En procédant à quelques découpages dans $X_{\max }$ nous arrivons à la Fig. 16. L'égalité des longueurs entre les $\gamma_{j}(j \in J=\{1, \ldots, 6\})$ entraîne que les coefficients de twist selon les géodésiques de $\Xi\left(\gamma_{1}\right)$ (dans la partition formée de ces géodésiques et de $\xi)$ valent $1 / 2$. Dans $X_{\max }$, nous avons deux triplets de géodésiques, $\left(\gamma_{5}, \gamma_{6}, \alpha_{1}\right)$ et $\left(\gamma_{3}, \gamma_{4}, \alpha_{2}\right)$, formant des triangles isocèles disjoints ; il existe une géodésique séparant $X_{\max }$ en deux tores à un bord contenant chacun un triangle, ces tores à un bord sont déterminés à isométries près par les longueurs $l\left(\gamma_{3}\right)=l\left(\gamma_{4}\right)=l\left(\gamma_{5}\right)=l\left(\gamma_{6}\right)$, il s'ensuit $l\left(\alpha_{1}\right)=l\left(\alpha_{2}\right)$. Enfin l'égalité entre la longueur de $\xi$ et celle des $\gamma_{j}(j \in J)$ détermine $X_{\max }$ de manière unique à isométrie près, $X_{\max } \simeq N(3)$ [15], mais $N(3) \notin$ $D \operatorname{car} \cosh (\xi / 2)<4,67$.

Dans la proposition qui suit nous faisons apparaître une configuration susceptible de produire un maximum, nous prouvons l'existence d'une surface réalisant cette configuration, et montrons sa maximalité vis-à-vis de la 3-systole.

Proposition 5.3 Dans $X_{\text {max }}$, trois géodésiques distinctes de $\left(\gamma_{j}\right)_{j \in J}$ ne passent jamais par un même point de Weierstrass. 


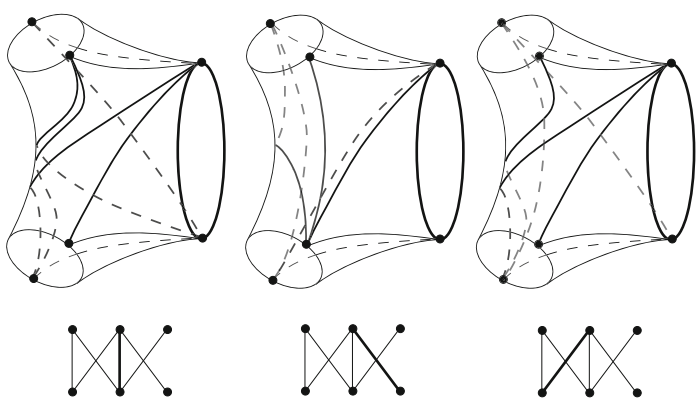

Fig. 17 Nouvelle configuration topologique

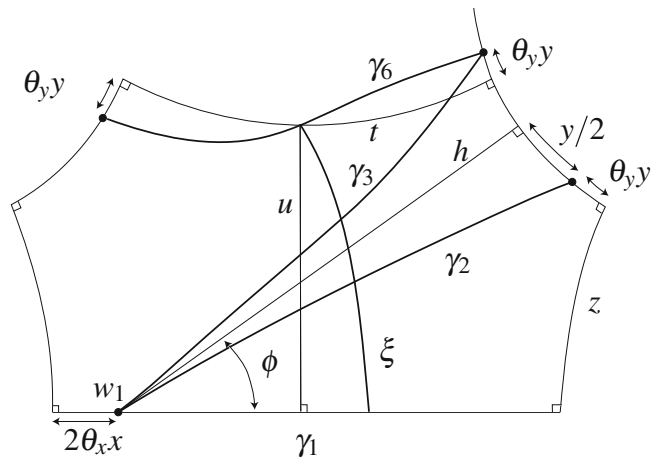

Fig. 18 Réalisation géométrique

Preuve Supposons que par un point de Weierstrass passent trois géodésiques de $\left(\gamma_{j}\right)_{j \in J}$. Découpons l'une de ces trois géodésiques ainsi que le couple qui lui est associé par $\Xi$. Nous obtenons deux pantalons hyperboliques, envoyés l'un sur l'autre par l'involution hyperelliptique. Il apparaît vite par les lemmes 5.1 et 5.2 qu'il n'y a que trois configurations topologiques possibles pour les $\left(\gamma_{j}\right)_{j \in J}$ dans ces pantalons (Fig. 17). En fait ces trois configurations correspondent à une seule et même configuration dans la surface toute entière, d'un point de vue combinatoire nous avons un seul et même graphe, il n'y a que l'arête découpée (en trait gras) qui change. Dans la suite de la preuve nous prendrons $J=\{1, \ldots, 6\}$.

Soient $w_{1}$ et $w_{4}$ les points de Weierstrass en lesquels concourent trois géodésiques de $\left(\gamma_{j}\right)_{j \in J}$, soit $\gamma_{1}$ la géodésique reliant $w_{1}$ et $w_{4}$, soient $\gamma_{2}$ et $\gamma_{3}$ les deux autres géodésiques passant par $w_{1}$, et soient $\gamma_{4}$ et $\gamma_{5}$ les deux autres géodésiques passant par $w_{4}$. Chacune des paires $\gamma_{2}, \gamma_{3}$ et $\gamma_{4}, \gamma_{5}$ forme un triangle isocèle avec une géodésique des $\alpha_{i}, \beta_{i}, \delta_{i}$, ces triangles sont disjoints et nous pouvons trouver une géodésique les séparant, par conséquent ils sont isométriques. Nous en déduisons que les deux bords différents de $\gamma_{1}$ dans le pantalon de gauche de la Fig. 17 ont même longueur. Vue la configuration des géodésiques $\gamma_{2}, \gamma_{3}, \gamma_{4}$ et $\gamma_{5}$ dans ce pantalon, nous avons nécessairement $\alpha_{1}=\alpha_{2}, \beta_{1}=\beta_{2}$ et $\delta_{1}=\delta_{2}$, en particulier les tores $\mathbb{T}_{1}$ et $\mathbb{T}_{2}$ sont isométriques. Notons que la translation d'ordre 2 selon $\gamma_{1}$ réalise un automorphisme de la surface $X_{\max }$. 
Nous allons prouver l'existence de la surface évoquée. En fait, il s'agit de réaliser la configuration de gauche de la Fig. 17. Nous considérons donc un pantalon avec deux bords égaux de longueur $2 y$, le troisième bord étant de longueur $4 x$. Nous exprimons trivialement les longueurs $t, u$ et $z$ (voir Fig. 18) en fonction de $x$ et $y$ :

$$
\begin{gathered}
\cosh ^{2} t=\frac{\cosh ^{2} x}{\cosh ^{2} y-1}+1, \\
\cosh ^{2} u=\frac{\cosh ^{2} y}{\cosh ^{2} x-1}+1, \\
\cosh ^{2} z=\frac{\cosh ^{2} x \cosh ^{2} y}{\left(\cosh ^{2} x-1\right)\left(\cosh ^{2} y-1\right)} .
\end{gathered}
$$

Sur chaque bord nous fixons deux points opposés correspondant aux points de Weierstrass, les couples sur les bords de longueur $2 y$ ont le même décalage $\theta_{y} y$ par rapport aux perpendiculaires communes. Les segments tracés en gras représentent les segments moitié des géodésiques $\gamma_{1}, \gamma_{2}, \gamma_{3}, \gamma_{6}$ et $\xi$. L'existence de la surface équivaut à l'existence de paramètres $x, \theta_{x}, y, \theta_{y}$ tels que les 5 segments géodésiques en gras soient de même longueur. Supposons $x$ et $y$ fixés, choisissons $\theta_{y}$ tel que $\gamma_{6}$ soit de longueur $4 x$, c'est-à-dire tel que $\cosh \left(\theta_{y} y\right)=\cosh x / \cosh t$; nous pouvons alors calculer $\sin \phi$ et $h$ en fonction de $x$ et $y$ :

$$
\begin{aligned}
\sin ^{2} \phi & =\cosh ^{2}\left(\frac{y}{2}+\theta_{y} y\right)+\cosh ^{2} z-\cosh ^{2}\left(\frac{y}{2}+\theta_{y} y\right) \cosh ^{2} z, \\
\cosh ^{2} h & =\frac{\cosh ^{2} z}{\sin ^{2} \phi},
\end{aligned}
$$

et en posant $X=\cosh x$ et $Y=\cosh y$ nous avons

$$
\begin{aligned}
\cosh ^{2}\left(\frac{y}{2}+\theta_{y} y\right)= & \frac{Y-1}{X^{2}+Y^{2}-1}\left[X^{2} Y^{2}+X^{2} Y-X^{2} / 2-Y^{2} / 2+1 / 2+\right. \\
& \left.X(Y+1) \sqrt{X^{2} Y^{2}-2 X^{2}-Y^{2}+1}\right],
\end{aligned}
$$

La condition $\gamma_{2}=\gamma_{3}=4 x$, équivalente à $\cosh h=\cosh (2 x) / \cosh (y / 2)$, donne implicitement $y$ en fonction de $x$. Nous élaborons facilement un petit programme qui en entrée prend $x$ et sort une valeur approchée de $y$. Pour différentes valeurs de $x$ nous calculons

$$
\begin{aligned}
\cosh ^{2}\left(2\left(1-\theta_{x}\right) x\right)= & \frac{X^{2}\left(X^{2}-1\right)}{X^{2}+Y^{2}-1}\left[8 X^{4}-12 X^{2}-4 Y^{2}+5\right. \\
& \left.-4\left(2 X^{2}-1\right) \sqrt{X^{4}-2 X^{2}-Y^{2}+1}\right]
\end{aligned}
$$

puis $\cosh ^{2}\left(2\left(1-\theta_{x}\right) x\right) \sin ^{2} \phi-\cosh ^{2}\left(y / 2+\theta_{y} y\right)$, cette grandeur prend des valeurs négatives et positives, nous en déduisons (en nous plaçant dans le trirectangle $h \gamma_{1} z y$ ) l'existence d'un $x$ tel que les géodésiques $\gamma_{1}, \gamma_{2}, \gamma_{3}, \gamma_{6}$ et $\xi$ sont de même longueur 
$4 x$. Nous trouvons que les coordonnées twists-longueurs solutions de notre problème sont approximativement

$$
\left(x ; \theta_{x} ; y ; \theta_{y}\right) \approx(1,1076 ; 0,17 ; 1,06 ; 0,15) .
$$

Nous venons de prouver l'existence d'une surface vérifiant la configuration voulue, on vérifie sa maximalité vis-à-vis de la systole de la famille $\mathcal{G}_{\xi} \cup \xi$ en calculant les gradients des fonctions longueur des systoles au point voulu (c'est un exercice de trigonométrie hyperbolique qui s'effectue sans difficultés avec l'aide de [8]). Nous observons que les conditions d'eutaxie et de perfection sont bien réalisées, l'analogue du théorème de Voronoï nous dit que cette surface est bien extrême pour la fonction sys $_{\mathcal{G}_{\xi} \cup \xi}$.

Il Reste à montrer que les systoles de $\mathcal{G}_{\xi} \cup \xi$ réalisent bien la 3 -systole de $X_{\text {max }}$. Les géodésiques de longueur $2 y$ sont les systoles de chacun des tores délimités par $\xi$, elles réalisent aussi la systole de $X_{\max }$. Nous repérons facilement la deuxième plus petite géodésique de ces tores. En comparant les longueurs de ces géodésiques avec celles des perpendiculaires communes du pantalon de la Fig. 18 nous voyons qu'elles réalisent chacune le rayon d'injectivité de la surface $X_{\max }$ en un point de Weierstrass, autrement dit elles induisent des arêtes de $G_{\text {cont }}\left(X_{\max }\right)$, et les géodésiques $\gamma_{1}, \ldots, \gamma_{6}$ réalisent la distance entre les deux groupes de points de Weierstrass. Considérons une partition de longueur minimale, elle contient au moins deux géodésiques non séparantes, nécessairement chacune d'elles est ou bien incluse dans l'un des tores délimités par $\xi$ ou bien un élément de la famille $\left\{\gamma_{1}, \ldots, \gamma_{6}\right\}$. Si l'une d'elles appartient à la famille $\left\{\gamma_{1}, \ldots, \gamma_{6}\right\}$ ces dernières réalisent bien la 3 -systole. Si les deux sont contenues dans un tore, alors la troisième géodésique de la partition est ou bien non séparante et dans ce cas appartient à la famille $\left\{\gamma_{1}, \ldots, \gamma_{6}\right\}$, ou bien séparante et dans ce cas c'est $\xi$ (il suffit de travailler un peu avec le rayon d'injectivité de $\xi$ ). Nous avons $\operatorname{sys}_{3}\left(X_{\text {max }}\right)=\operatorname{sys}_{\mathcal{F}}\left(X_{\text {max }}\right) \approx 2 \operatorname{arccosh}(4,63)$.

\subsubsection{Conclusion}

Proposition 5.4 Les points de D réalisant un maximum local de la systole de $\mathcal{F}$ sont ceux correspondant à la surface $P(3)$ marquée.

Preuve Pour que le point $M_{\max }$ soit un maximum local de la fonction sys ${ }_{\mathcal{F}}$ sur $D$, la famille $\left(\gamma_{j}\right)_{j \in J}$ doit contenir au moins 6 éléments. Les propositions 5.2 et 5.3 nous éclairent sur la configuration des $\gamma_{j}(j \in J)$, via des découpages nous constatons qu'il y a une unique configuration (combinatoire, topologique) possible (Fig. 19). Cette configuration est exactement celle des géodésiques de $P(3)$ réalisant la systole de $\mathcal{F}$, or nous savons qu' une configuration est réalisée par au plus une surface extrême. Donc $X_{\max }$ est isométrique à $P(3)$.

Théorème 5.1 La 3-systole atteint son maximum global sur $\mathcal{T}_{2}$ aux points correspondant à la surface $P(3)$ marquée.

La géodésique $\xi$ sépare $P(3)$ en deux tores équilatéraux, la longueur du rayon minimal de ces tores est donnée par (3.2), et le coefficient de twist $\theta_{\xi}$ vaut $1 / 12$. En 


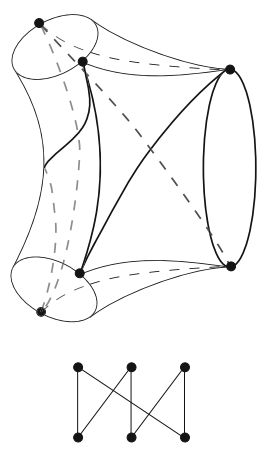

Fig. 19 Configuration topologique de $P(3)$

injectant tout ceci dans la formule du $§ 2.4$ nous obtenons :

$$
\cosh (B(2) / 2)=\frac{1}{4\left[\cosh ^{2}(B(2) / 12)-1\right]}+\cosh (B(2) / 12)
$$

Il vient que $\cosh (B(2) / 12)$ est solution de $32 X^{5}-32 X^{4}-24 X^{3}+24 X^{2}-1=0$, cela donne $\cosh (B(2) / 2) \approx 4,67096$.

\subsection{Commentaire}

La méthode introduite semble assez efficace. En effet, en étudiant les différentes configurations topologiques susceptibles de réaliser un maximum de la systole de la famille $\mathcal{G}_{\xi} \cup\{\xi\}$, nous avons naturellement retrouvé les deux surfaces $P(3)$ et $N(3)$ découvertes par Schmutz Schaller; nous avons de plus fait apparaître un troisième maximum local de la 3-systole, infirmant ainsi la conjecture de Schmutz Schaller, à savoir que les seuls maxima de la 3 -systole sur l'espace de Teichmüller $\mathcal{T}_{2}$ sont les points associés aux surfaces $N(3)$ et $P(3)$.

Remerciements Cet article correspond à la deuxième partie de ma thèse de doctorat, je tiens à remercier mon directeur Christophe Bavard pour son soutient et sa disponibilité. Ce travail a commencé au centre Bernoulli de l'EPFL lors du semestre Spaces of Negative Curvature, je remercie les organisateurs pour leur accueil.

\section{Bibliographie}

1. Bavard, C.: La Systole des Surfaces Hyperelliptiques. Prépublication de 1'ENS Lyon no. 71, Juillet (1992)

2. Bavard, C.: Disques extrémaux et surfaces modulaires. Ann. Fac. Sci. Toulouse Math. (6) 5(2), 191-202 (1996)

3. Bavard, C.: Systole et invariant d'Hermite. J. Reine Angew. Math. 482, 93-120 (1997)

4. Bavard, C.: Anneaux extrémaux dans les surfaces de Riemann. Manuscripta Math. 117, 265-271 (2005)

5. Bavard, C.: Théorie de Voronoï géométrique. Propriétés de finitude pour les familles de réseaux et analogues. Bull. Soc. Math. France 133(2), 205-257 (2005) 
6. Bers, L.: Spaces of degenerating Riemann surfaces. In: Discontinuous groups and Riemann surfaces (Proceedings of Conference, University of Maryland, College Park, Md., 1973). Ann. of Math. Studies, No. 79, pp. 43-55. Princeton University Press (1974)

7. Böröczky, K.: Packing of spheres in spaces of constant curvature. Acta Math. Acad. Sci. Hungar. 32(3-4), 243-261 (1978)

8. Buser, P.: Geometry and Spectra of Compact Riemann Surfaces, Progress in Mathematics, vol. 106. Birkhäuser, Switzerland (1992)

9. Buser, P., Seppälä, M.: Symmetric pants decompositions of Riemann surfaces. Duke Math. J. 67(1), 39-55 (1992)

10. Diestel, R.: Graph Theory, Graduate Texts in Mathematics, vol. 173, 4th edn. Springer, Berlin (2005)

11. Gendulphe, M.: Paysage systolique des surfaces hyperboliques compactes de caractéristique -1 . disponible à http://matthieu.gendulphe.com

12. Mumford, D.: A remark on Mahler's compactness theorem. Proc. Amer. Math. Soc. 28, 289-294 (1971)

13. Parlier, H.: On the geometry of simple closed geodesics. PhD thesis, EPFL (2004)

14. Parlier, H.: Lengths of geodesics on Riemann surfaces with boundary. Ann. Acad. Sci. Fenn. Math. 30(2), 227-236 (2005)

15. Schmutz, P.: Riemann surfaces with shortest geodesic of maximal length. Geom. Funct. Anal. 3(6), 564-631 (1993) 